\title{
Robust pricing and hedging under trading restrictions and the emergence of local martingale models
}

\author{
Alexander M.G. Cox ${ }^{1}$ Zhaoxu Hou' ZJan Obłój $^{2}$
}

Received: 13 August 2014 / Accepted: 22 July 2015 / Published online: 17 March 2016

(C) The Author(s) 2016. This article is published with open access at Springerlink.com

\begin{abstract}
We pursue the robust approach to pricing and hedging in which no probability measure is fixed, but call or put options with different maturities and strikes can be traded initially at their market prices. We allow the inclusion of robust modelling assumptions by specifying a set of feasible paths on which (super)hedging arguments are required to work. In a discrete-time setup with no short selling, we characterise absence of arbitrage and show that if call options are traded, then the usual pricinghedging duality is preserved. In contrast, if only put options are traded, a duality gap may appear. Embedding the results into a continuous-time framework, we show that the duality gap may be interpreted as a financial bubble and link it to strict local martingales. This provides an intrinsic justification of strict local martingales as models for financial bubbles arising from a combination of trading restrictions and current market prices.
\end{abstract}

Keywords Robust pricing and hedging · Financial bubble $\cdot$ Local martingale models · Pricing-hedging duality · Trading restrictions $\cdot$ Short selling constraint · Martingale optimal transport

Mathematics Subject Classification (2010) 91G20 • 91B24 • 60G42 · 60G44

JEL Classification $\mathrm{C} 61 \cdot \mathrm{G} 13 \cdot \mathrm{E} 32$

J. Obłój

jan.obloj@maths.ox.ac.uk

A.M.G. Cox

A.M.G.Cox@bath.ac.uk

Z. Hou

zhaoxu.hou@maths.ox.ac.uk

1 Department of Mathematical Sciences, University of Bath, Bath BA2 7AY, UK

2 Mathematical Institute, University of Oxford, ROQ, Woodstock Road, Oxford OX2 6GG, UK 


\section{Introduction}

The approach to pricing options through considering the dual problem of finding the expected value of the payoff under a risk-neutral measure is both classical and well understood. In a complete market setting, it is simply the way to compute the hedging price, as argued by Black and Scholes [5]. In incomplete markets, optimising over risk-neutral expectations allows the computation of the superhedging price, as argued by El Karoui and Quenez [17] and in the seminal work of Delbaen and Schachermayer [15]. Almost as classical is the problem of finding superhedging prices under various constraints on the set of admissible portfolios. Questions of this type arise in Cvitanić and Karatzas [12], where convex constraints in the hedging problem lead to a dual problem where one looks for the largest expectation of the payoff of the derivative in a class of auxiliary markets, where the auxiliary markets are a modification of the original markets reflecting the trading constraints. In the special case of markets where participants may not short sell assets, the class of auxiliary markets corresponds to the class of supermartingale measures. (For further results in this direction, see e.g. Jouini and Kallal [30], Cvitanić et al. [13], Pham and Touzi [35], Pulido [37].)

In this paper, we combine trading constraints with concepts from robust derivative pricing. In robust pricing, one aims to minimise modelling assumptions by not pre-supposing the existence of a given probabilistic model for the underlying assets. Instead, we replace modelling assumptions by two weaker assumptions: first, we suppose that our observed realisation of the price process will lie in some set $\mathfrak{P}$ of possible outcomes, e.g. the set of paths whose sum of squared differences is bounded by some given constant, or the continuous-time analogue, the set of paths with quadratic variation bounded by a given constant; second, we suppose that there are additional options which may be traded at time zero, for prices which are observed in the market. In this paper, we assume that the additional options which are traded are either European put or call options. In particular, we suppose that at fixed maturity dates, the prices of all puts/calls on the underlying are known. The presence of put and call options fixes the law of the process under the risk-neutral measure in any calibrated model - this is a fact first observed by Breeden and Litzenberger [7] and hence constrains the set of probability measures over which we optimise. There has recently been substantial interest in robust pricing problems with a literature that can be traced back to the seminal paper of Hobson [24]. The results in this paper are based on the discrete-time approach of Beiglböck et al. [4], where a duality result is shown using concepts from optimal transport.

In the discrete-time setting, our results can be summarised as follows. We suppose we are given a sequence of call price functions at maturity dates $T_{1}<T_{2}<\cdots<T_{n}$. We show that these prices are consistent with the absence of (suitably defined) arbitrage opportunities if and only if they give rise to a sequence of probability measures $\mu_{1}, \ldots, \mu_{n}$ on $\mathbb{R}_{+}$which satisfy natural ordering properties. These then, as explained above, correspond to the implied marginal distributions of the asset under feasible risk-neutral measures. (Note that here and throughout, we assume that all assets are denominated in units of some numeraire, for example discounted by the money market account.) Classically, the measures would be in convex order. However, in the 
absence of the ability to short sell the asset, it is not possible to generate an arbitrage when $m_{k}=\int x \mu_{k}(\mathrm{~d} x)>\int x \mu_{k+1}(\mathrm{~d} x)=m_{k+1}$, and so the expected value of the asset according to the (implied) risk-neutral measure may be smaller at later maturities. We then show that the minimal price of a portfolio involving call options and long positions in the asset, and which superhedges a derivative for every path in $\mathfrak{P}$, is equal to the supremum of the expected value of the derivative's payoff, where the supremum is taken over all supermartingale measures which have full support on $\mathfrak{P}$, and under which the law of the asset at $T_{k}$ is equal to $\mu_{k}$. This result generalises Corollary 1.1 in Beiglböck et al. [4] by including a restriction to a certain set of paths $\mathfrak{P}$ and a short selling constraint. Observe also that in the case where the measures $\mu_{k}$ all have the same mean, which is equal to the initial stock price $s_{0}$, the class of supermartingale measures is simply the class of martingale measures.

We also consider the case where the set of call options is replaced by put options with the same maturities. Since short selling of the asset is not permitted, one cannot immediately compare to the case where the call options are available to trade, even if the set of possible implied marginal laws remains the same. In this case, we show that a duality gap arises when the initial asset price $s_{0}$ is strictly larger than the implied mean $m_{k}$ for some maturity $T_{k}$. In particular, there is no longer equality between the cheapest superhedge and the largest model-consistent price-rather, we see a difference which can be characterised in terms of the limit behaviour of the put prices as the strike goes to infinity.

The easiest example of this duality gap arises in considering the difference between the implied price of a forward contract written on the asset-if we take the forward to be a contract which pays the holder the value of the asset at some future date $T_{k}$, then the forward contract will have a model-implied price $m_{k}=\int x \mu_{k}(\mathrm{~d} x)$, which in the cases of interest will be strictly smaller than the initial price of the asset $s_{0}$. In the case where call options are traded, the forward may be superhedged for $m_{k}$ using call options (the call option with strike 0 has the same payoff as the forward). In the case where put options are traded, this is not the case-instead, the cheapest superreplicating strategy will simply be to purchase the asset at time 0 , which has cost $s_{0}$.

Historically, there has been relatively little study in the literature of asset prices which are strict supermartingales ${ }^{1}$ under the risk-neutral measure. Their main appearance has been as models for the study of financial bubbles, where strict local martingales are considered. We believe that our results, both in discrete time and in continuous time, contribute to and provide a novel perspective on the existing literature on financial bubbles.

In mathematical finance, the modelling of financial bubbles using local martingale models can be traced back to Heston et al. [23], with subsequent contributions including Cox and Hobson [10], Jarrow et al. [28, 29]. Our work also has interesting parallels to the recent work of Herdegen and Schweizer [22]. Before Heston et al. [23], a number of authors observed that in certain circumstances, models

\footnotetext{
${ }^{1}$ A strict supermartingale is a supermartingale which is not a martingale. Since we only consider nonnegative processes over finite time horizons, a strict supermartingale is therefore a supermartingale which has a non-constant expected value. Similarly, a strict nonnegative local martingale is a local martingale which is also a strict supermartingale.
} 
which were only strict local martingales arise naturally and/or are interesting of their own right (and can be attributed some financial interpretation); see Lewis [31], Delbaen and Schachermayer [16], Loewenstein and Willard [32], Sin [39]. One of the most common examples of a naturally occurring class of local martingale models is the class of CEV models, $d S_{t}=S_{t}^{\alpha} d B_{t}, S_{0}=s_{0}$, where $\alpha>1$. In the case where $\alpha=2$, one recovers the inverse of a 3-dimensional Bessel process, which was studied in Delbaen and Schachermayer [16]. More recently, quadratic normal volatility (QNV) models have also been studied, which are mostly strict local martingales, but typically calibrate well to market data; see Carr et al. [9].

We build our contribution to this literature by embedding the discrete-time results into a continuous-time framework. Consider a continuous-time market with dynamic trading in the asset and call or put options traded initially for certain fixed maturities. Then the discrete setup is naturally included by considering trading strategies which only rebalance at the maturity dates of the options. Discrete-time supermartingale measures are obtained as projections of local martingale measures which meet the given marginals. The duality gap is preserved when put options trade, and this gap has a possible interpretation as a financial bubble. To make this generalisation, it is necessary to introduce a pathwise superhedging requirement which enforces a collateral requirement. A similar requirement has already been considered in Cox and Hobson [10]. We therefore believe that an important consequence of this paper is the following interpretation of local martingale models in financial applications: local martingale models naturally arise due to trading constraints.

This has an impact on the existing literature on financial bubbles: intrinsically, we believe that models where asset prices are strict local martingales (under a risk-neutral measure) are models which arise due to constraints on possible trading strategies. They thus correspond exactly to rational or speculative bubbles in the asset pricing and economics literature. These are usually driven by short selling constraints and/or disagreement between the agents on the fundamental values due to heterogenous beliefs or overconfidence; see Hugonnier [26], Harrison and Kreps [20], and Scheinkman and Xiong [38]. Strict local martingale models are a very natural class of models for bubbles, since there is a natural notion of a 'fundamental' price which diverges from the traded price. However, as we show, this divergence is 'rational' and driven by the absence of arbitrage combined with trading restrictions, as in speculative bubbles. This is different from the case of an 'irrational bubble' when divergence between the market price of an asset and its fundamental price is driven by some behavioural aspect of market participants, rather than specific market features. In this sense, an important contribution of this article for the literature on bubbles is to divorce any notions of 'irrationality' from the financial study of strict local martingale models.

We also make the observation that although we present results on local martingale models in continuous time, our approach is firmly rooted in a discrete-time setup, and all pricing results in continuous time follow essentially from the corresponding discrete-time results. One interpretation is that these models therefore are the natural discrete-time analogues of local martingale models (in this sense, our results provide an alternative response to the criticism that local martingale models for bubbles are only a feature of continuous-time models, as discussed in Protter [36, Sect. 11]; see 
also Jarrow and Protter [27]). However, it seems to us that the implication more naturally runs in the other direction: in discrete time, our models are very natural and easily specified. In continuous time, however, local martingales are very subtle processes, and the difference between a local martingale and a martingale is not easy to detect-our paper provides a clear specification of a discrete-time setup which could be interpreted in continuous time as a local martingale model. As a result, in our setup, local martingale phenomena arise naturally, and reflect specific market conditions. This contrasts with the arguments of e.g. Guasoni and Rasonyi [19], who argue against local martingale models on the basis that they can always be approximated by martingale models.

Short selling bans as a regulatory tool to discourage speculation and stabilise markets have proved to be popular among emerging markets and during times of financial crisis. During the US subprime mortgage crisis, short selling of 797 financial stocks in US markets was banned by the SEC between September 19, 2008 and October 8, 2008. Around the same time, the South Korean Financial Supervisory Commission imposed an outright prohibition of short selling of any listed stocks in an attempt to curb the spread of malignant rumours in the market. The ban was lifted for nonfinancial stocks about a year later, while the constraints on financial stocks remained until November 2013. Interestingly, the US and South Korea both have very active derivatives markets, and in both examples, the bans on short selling did not extend to derivative markets. This allowed market makers and investors to use options to hedge portfolios and express pessimistic views. In light of a series of short selling bans across the globe, the question of their impact on stocks and derivatives markets is once again a matter of concern to academics and policy makers; see e.g. Battalio and Schultz [2], Hendershott et al. [21]. The current paper thus represents a theoretical contribution to this literature. Battalio and Schultz [2] study the US short selling ban in September 2008 and find that synthetic share prices for banned stocks, computed separately for puts and for calls, become significantly lower than the actual share prices, accompanied by increases in bid-ask spreads. The findings correspond to the setting of our paper with $m_{k}<s_{0}$, making it particularly interesting.

Finally, we note that in parallel to our research, Fahim and Huang [18] and Bayraktar and Zhou [3] considered discrete-time robust pricing and hedging with trading restrictions. Fahim and Huang [18] use concepts from optimal martingale transport but assume a market input in the form of distributions $\mu_{i}$ already satisfying a set of assumptions which in our paper are characterised in terms of arbitrage opportunities. Bayraktar and Zhou [3] adopt the quasi-sure analysis of Bouchard and Nutz [6] with finitely many traded options. As a result, in both cases the pricing-hedging duality holds and no links are made to modelling of financial bubbles in discrete or continuous time. The focus of both papers is on general convex portfolio constraints.

This paper is organised as follows. Section 2 discusses the robust modelling framework in discrete time. Sections 3 and 4 specialise respectively to the case when call or put options are traded. The latter in particular explores when a duality gap arises. Subsequently, Sect. 5 focuses on the continuous-time setup. Several proofs are relegated to the Appendix. 


\section{Robust framework for pricing and hedging}

We consider a financial market with two assets: a risky asset $S$ and a numeraire (e.g. the money market account). All prices are denominated in the units of the numeraire. In particular, the numeraire's price is thus normalised and equal to one. We assume initially that $S$ is traded discretely in time at dates $0=T_{0}<T_{1}<T_{2}<\cdots<T_{n}=T$. This is extended to a continuous-time setup in Sect. 5. The asset starts at $S_{0}=s_{0}>0$ and is assumed to be nonnegative. We work on the canonical space with a fixed starting point $\Omega=\left\{\left(\omega_{0}, \ldots, \omega_{n}\right) \in \mathbb{R}_{+}^{n+1}: \omega_{0}=s_{0}\right\}$. The coordinate process on $\Omega$ is denoted by $\mathbb{S}=\left(\mathbb{S}_{i}\right)_{i=0}^{n}$, i.e.,

$$
\mathbb{S}_{i}: \Omega \rightarrow \mathbb{R}_{+}, \mathbb{S}_{i}\left(\omega_{0}, \omega_{1}, \ldots, \omega_{n}\right)=\omega_{i}, \quad i=0, \ldots, n
$$

and $\mathbb{F}=\left(\mathcal{F}_{i}\right)_{i=0}^{n}$ is its natural filtration, $\mathcal{F}_{i}=\sigma\left(\mathbb{S}_{0}, \ldots, \mathbb{S}_{i}\right)$ for any $i=0, \ldots, n$.

We pursue here a robust approach and do not postulate any probability measure which would specify the dynamics for $S$. Instead we assume that there is a set $\mathcal{X}$ of market-traded options with prices $\mathcal{P}(X), X \in \mathcal{X}$, known at time zero. The trading is frictionless, and options in $\mathcal{X}$ may be bought or sold at time zero at their known prices. Hence we extend $\mathcal{P}$ to a linear operator defined on

$$
\operatorname{Lin}(\mathcal{X})=\left\{a_{0}+\sum_{i=1}^{m} a_{i} X_{i}: m \in \mathbb{N}, a_{0}, a_{i} \in \mathbb{R}, X_{i} \in \mathcal{X} \text { for all } i=1, \ldots, m\right\}
$$

As explained above, the numeraire has a constant price equal to one. Finally, the risky asset $S$ may be traded at any $T_{i}, i=0, \ldots, n$; however, short selling is not allowed.

We consider two cases, namely when $\mathcal{X}$ is composed of call options or of put options:

$$
\begin{aligned}
& \mathcal{X}_{c}=\left\{\left(\mathbb{S}_{i}-K\right)^{+}: i=1, \ldots, n, K \in \mathbb{R}_{+}\right\} \\
& \mathcal{X}_{p}=\left\{\left(K-\mathbb{S}_{i}\right)^{+}: i=1, \ldots, n, K \in \mathbb{R}_{+}\right\}
\end{aligned}
$$

An admissible (semi-static) trading strategy is a pair $(X, \Delta)$, where $X \in \operatorname{Lin}(\mathcal{X})$ and $\Delta=\left(\Delta_{j}\right)$ are bounded nonnegative measurable functions $\Delta_{j}: \mathbb{R}_{+}^{j} \rightarrow \mathbb{R}_{+}$, $j=0, \ldots, n-1$. Here and throughout, $\mathbb{R}_{+}^{0}:=\{0\}$, which simply means that $\Delta_{0}$ is a nonnegative constant. The total payoff associated to $(X, \Delta)$ is given by

$$
\Psi_{X, \Delta}(\mathbb{S}):=X(\mathbb{S})+\sum_{j=0}^{n-1} \Delta_{j}\left(\mathbb{S}_{1}, \ldots, \mathbb{S}_{j}\right)\left(\mathbb{S}_{j+1}-\mathbb{S}_{j}\right)
$$

The cost of following such a trading strategy is equal to the cost of setting up its static part, i.e., of buying the options at time zero, and is equal to $\mathcal{P}(X)$. We denote the class of admissible (semi-static) trading strategies by $\mathcal{A}_{\mathcal{X}}$. We write $\mathcal{A}_{c}$ (resp., $\mathcal{A}_{p}$ ) for the case $\mathcal{X}=\mathcal{X}_{c}$ (resp., $\mathcal{X}=\mathcal{X}_{p}$ ). Note that since short selling is not allowed, these are genuinely different and, as we shall see, give very different results. Indeed, note that in the former, the short selling of call options is allowed, including the strike zero, i.e., 
the forward, providing a superreplication of the asset $S$, possibly at a strictly cheaper price than $s_{0}$. This feature is not present when dealing with put options.

We are interested in characterising and computing superhedging prices. All the quantities we have introduced are defined pathwise and the superhedging property is also required to hold pathwise. We have also made only mild assumptions on the market mechanisms (e.g. no frictions), but no specific modelling assumptions on the dynamics of the assets. A natural way to incorporate beliefs into the robust framework is through specifying the set $\mathfrak{P} \subset \Omega$ of "possible paths", i.e., paths we deem feasible and for which the hedging strategies are required to work. This can be thought of as specifying the maximal support of the plausible models. In this way, with the support ranging from all paths to e.g. paths in a binomial model, the robust framework can interpolate between model-independent and model-specific setups. The set $\mathfrak{P}$ might be obtained through time series analysis of past data combined with modelling and a given agent's idiosyncratic views, and is referred to as the prediction set. Note that since there is no probability measure specified and hence no distinction between the real and the risk-neutral measure, it is very natural to combine two streams of information: time series of past data and forward-looking option prices. This idea goes back to [34] and we refer to Hou and Obłój [25] and Spoida [40] for more details and extended discussion.

We call the triplet $(\mathcal{X}, \mathcal{P}, \mathfrak{P})$ of market traded options $\mathcal{X}$, their prices $\mathcal{P}$ and prediction set $\mathfrak{P}$ the robust modelling input. The fundamental financial notions defined below, e.g. arbitrage or the superreplication price, are implicitly relative to these inputs.

Definition 2.1 The superreplication cost of a derivative given by a payoff function $G: \Omega \rightarrow \mathbb{R}$, denoted by $V_{\mathcal{X}, \mathcal{P}, \mathfrak{P}}(G)$, is the smallest initial capital required to finance an admissible semi-static trading strategy which superreplicates $G$ for every path in $\mathfrak{P}$, i.e.,

$$
V_{\mathcal{X}, \mathcal{P}, \mathfrak{P}}(G):=\inf \left\{\mathcal{P}(X): \exists(X, \Delta) \in \mathcal{A}_{\mathcal{X}} \text { such that } \Psi_{X, \Delta} \geq G \text { on } \mathfrak{P}\right\} .
$$

Note that since $\omega_{0}=s_{0}$ for all $\omega \in \Omega$, it is equivalent to see $G$ as a function from $\Omega$ or from $\mathbb{R}_{+}^{n}$. We shall be tacitly switching between these viewpoints; the former is used when writing $G=G(\mathbb{S})$, the latter when imposing conditions on $G$, see e.g. (3.1) below.

Our aim is to understand when a pricing-hedging duality holds, i.e., when the superreplication price can be computed through the supremum of expectations of the payoff over a suitable class of probabilistic models.

Definition 2.2 A market-calibrated model is a probability measure $\mathbb{P}$ on $(\Omega, \mathbb{F})$ satisfying $\mathbb{P}[\mathfrak{P}]=1$ and for any $(X, \Delta) \in \mathcal{A}_{\mathcal{X}}$

$$
\mathbb{E}_{\mathbb{P}}\left[\Psi_{X, \Delta}(\mathbb{S})\right] \leq \mathcal{P}(X),
$$

where here and throughout, we make the convention that $\infty-\infty=-\infty$ so that the left-hand side (LHS) in (2.1) is always well defined. The set of market-calibrated models is denoted by $\mathcal{M}_{\mathcal{X}, \mathcal{P}, \mathfrak{P}}^{-}$. 
Remark 2.3 It follows from the definition that if $\mathcal{M}_{\mathcal{X}, \mathcal{P}, \mathfrak{P}}^{-} \neq \emptyset$, then for any Borel function $G: \Omega \rightarrow \mathbb{R}$,

$$
P_{\mathcal{X}, \mathcal{P}, \mathfrak{P}}(G):=\sup _{\mathbb{P} \in \mathcal{M}_{\mathcal{X}, \mathcal{P}, \mathfrak{P}}^{-}} \mathbb{E}_{\mathbb{P}}[G(\mathbb{S})] \leq V_{\mathcal{X}, \mathcal{P}, \mathfrak{P}}(G)
$$

We sometimes refer to the LHS of the above inequality as the primal value and to the RHS as the dual value. This convention is borrowed from the literature on the martingale optimal transport problem. Both sides of (2.2) could be interpreted as a notion of "price" of the asset. The superhedging price $V_{\mathcal{X}, \mathcal{P}, \mathfrak{P}}(G)$ arises from (efficient) trading. The primal value $P_{\mathcal{X}, \mathcal{P}, \mathfrak{P}}(G)$ arises from modelling. Indeed, it represents the worst model price, among models consistent with the prices observed in the market, and can be thought of as the fundamental price of $G$. We also emphasise that either or both of these may be different from the market prices, which are observable and equal to $s_{0}$ for the dynamically traded asset and to $\mathcal{P}(X)$ for $X \in \mathcal{X}$. The case of strict inequality in (2.2) admits an interpretation as a financial bubble-an issue we consider in detail further below.

Remark 2.4 It follows from the definition that under any market-calibrated model $\mathbb{P}$, the canonical process $\mathbb{S}=\left(\mathbb{S}_{i}\right)_{i=1}^{n}$ is a supermartingale. Such a measure is called a supermartingale measure. Furthermore, for any $X \in \mathcal{X},(2.1)$ holds for both $(X, 0)$ and $(-X, 0)$ so that $\mathbb{P}$ is calibrated to options in $\mathcal{X}$, i.e., $\mathbb{P}$ satisfies $\mathbb{E}_{\mathbb{P}}[X]=\mathcal{P}(X)$ for any $X \in \mathcal{X}$.

Definition 2.5 We say there is a robust uniformly strong arbitrage if there exists a trading strategy $(X, \Delta) \in \mathcal{A}_{\mathcal{X}}$ with a negative price $\mathcal{P}(X)<0$ and a nonnegative payoff $\Psi_{X, \Delta}(\mathbf{s}) \geq 0$ for all $\mathbf{s} \in \mathfrak{P}$.

Remark 2.6 By definition, it is clear that the market admits a robust uniformly strong arbitrage if and only if $V_{\mathcal{X}, \mathcal{P}, \mathfrak{P}}(0)<0$. When $\mathfrak{P}=\Omega$, the notion of robust uniformly strong arbitrage corresponds to a model-independent arbitrage; see Davis and Hobson [14] and Cox and Obłój [11]. In a general robust setting, the existence of an arbitrage in the above sense may depend on the modelling assumptions, expressed through $\mathfrak{P} \subset \Omega$, which justifies the terminology. Equally, we stress that the arbitrage is required to be uniform in outcomes $\mathbf{s} \in \mathfrak{P}$ to distinguish from a slightly weaker notion, used in Acciaio et al. [1], of a strategy $(X, \Delta) \in \mathcal{A}_{\mathcal{X}}$ with $\mathcal{P}(X) \leq 0$ and a positive payoff $\Psi_{X, \Delta}(\mathbf{s})>0$, for all $\mathbf{s} \in \mathfrak{P}$. We refer to the latter as robust strong arbitrage. The two notions are not equivalent in general. However, we can show that they are equivalent in our setup when $\mathcal{X}=\mathcal{X}_{p}$. Likewise, when $\mathcal{X}=\mathcal{X}_{c}$, we can show they are equivalent when either $\mathfrak{P}=\Omega$ or property (iii) in Condition 3.1 below holds.

The three papers mentioned in the above remark also show that typically absence of robust uniformly strong arbitrage is not sufficient to guarantee that a (robust) fundamental theorem of asset pricing holds, and introduce various weaker notions or additional assumptions. Here we follow Cox and Obłój [11]: 
Definition 2.7 We say that there is a weak free lunch with vanishing risk (WFLVR) if there exist admissible trading strategies $\left(X_{k}, \Delta_{k}\right) \in \mathcal{A}_{\mathcal{X}}$ and $(X, \Delta) \in \mathcal{A}_{\mathcal{X}}$ such that $\Psi_{X_{k}, \Delta_{k}} \rightarrow 0$ pointwise on $\mathfrak{P}, \lim _{k} \mathcal{P}\left(X_{k}\right)$ is well defined with $\lim _{k} \mathcal{P}\left(X_{k}\right)<0$ and $\Psi_{X_{k}, \Delta_{k}} \geq \Psi_{X, \Delta}$.

Note that the requirement that $\lim _{k} \mathcal{P}\left(X_{k}\right)$ exists is made with no loss of generality as we could always pass to a subsequence of strategies. Note also that a robust uniformly strong arbitrage is by definition also a WFLVR. A version of the fundamental theorem of asset pricing in our context, given below in Proposition 3.2 for the case $\mathcal{X}=\mathcal{X}_{c}$ and in Proposition 4.2 for the case $\mathcal{X}=\mathcal{X}_{p}$, states that absence of WFLVR is equivalent to existence of a market-calibrated model. Further, as in Davis and Hobson [14] and Cox and Obłój [11], we can characterise absence of WFLVR through the properties of $\mathcal{P}$. This is insightful and is one of the reasons why we prefer to keep the option payoffs fixed, e.g. call options in $\mathcal{X}_{c}$, and discuss their prices $\mathcal{P}$, as opposed to considering shifted payoffs $X-\mathcal{P}(X)$ and eliminating $\mathcal{P}$ from the discussion as in e.g. Acciaio et al. [1].

\section{Robust pricing-hedging duality when call options trade}

In this section, we consider the market in which call options are traded, i.e., $\mathcal{X}=\mathcal{X}_{c}$. Our main result states that we recover the duality known from the case when short selling restrictions are not present. Throughout we assume that $\mathfrak{P}$ is a closed subset of $\Omega$.

\subsection{Market input and no arbitrage}

We start by establishing a robust fundamental theorem of asset pricing for our setting which links absence of arbitrage, properties of call prices, and existence of a marketcalibrated model.

Condition 3.1 Let $\mathcal{X}=\mathcal{X}_{c}$ and $c_{i}(K):=\mathcal{P}\left(\left(\mathbb{S}_{i}-K\right)^{+}\right), i=1, \ldots, n, K \geq 0$. Then

(i) $c_{i}(x)$ is a nonnegative, convex, decreasing function of $x$ on $\mathbb{R}_{+}$;

(ii) $s_{0} \geq c_{1}(0) \geq \cdots \geq c_{n}(0) \geq 0$ and $c_{i}^{\prime}(0+) \geq-1$;

(iii) $c_{i}(K) \rightarrow 0$ as $K \rightarrow \infty$;

(iv) for any $x \in \mathbb{R}_{+}, c_{i}(0)-c_{i}(x)$ is nonincreasing in $i$.

A robust fundamental theorem of asset pricing in our setup reads as follows.

Proposition 3.2 Suppose $\mathfrak{P}$ is a closed subset of $\Omega$ and $\mathcal{X}=\mathcal{X}_{c}$. Then there is no WFLVR if and only if there exists a market-calibrated model, which then implies that Condition 3.1 holds. Furthermore, if $\mathfrak{P}=\Omega$, then Condition 3.1 implies the absence of WFLVR.

Proposition 3.3 Suppose $\mathfrak{P}=\Omega$ and $\mathcal{X}=\mathcal{X}_{c}$. Then Condition 3.1(i), (ii) and (iv) are necessary and sufficient for the absence of robust uniformly strong arbitrage. 
In consequence, when these conditions hold but Condition 3.1(iii) fails, there is no robust uniformly strong arbitrage, but a market-calibrated model does not exist.

We defer the proofs of Propositions 3.2 and 3.3 to Sects. 6.2 and 6.4.

Remark 3.4 If we assume that there is no robust uniformly strong arbitrage, then we can immediately deduce that $c_{i}(0)$ is nonincreasing in $i$. Indeed, if there exists some $i$ such that $c_{i}(0)<c_{i+1}(0)$, then by taking $\Delta_{i}=1, \Delta_{j}=0$ for $j \neq i$ and $X=\left(\mathbb{S}_{i}-0\right)^{+}-\left(\mathbb{S}_{i+1}-0\right)^{+}$, we have $\mathcal{P}(X)=c_{i}(0)-c_{i+1}(0)<0$ but $\Psi_{X, \Delta}=0$ which shows that $(X, \Delta)$ is a robust uniformly strong arbitrage.

\subsection{Robust pricing-hedging duality and (super-)martingale optimal transport}

Our main theorem in Sect. 3 states that the pricing-hedging duality is preserved under a ban on short selling when call options are traded.

Theorem 3.5 Suppose that the market input $\left(\mathcal{X}_{c}, \mathcal{P}, \mathfrak{P}\right)$ admits no WFLVR. Let $G: \mathbb{R}_{+}^{n} \rightarrow[-\infty, \infty)$ be an upper semi-continuous function such that

$$
G\left(s_{1}, \ldots, s_{n}\right) \leq K\left(1+s_{1}+\cdots+s_{n}\right)
$$

on $\mathbb{R}_{+}^{n}$ for some constant $K$. Then the pricing-hedging duality holds, i.e.,

$$
P_{\mathcal{X}_{c}, \mathcal{P}, \mathfrak{P}}(G)=V_{\mathcal{X}_{c}, \mathcal{P}, \mathfrak{P}}(G)
$$

Remark 3.6 Our proof of this result closely follows Beiglböck et al. [4] and is an application of the duality theory from optimal transport, which allows us to express the dual problem as a min-max calculus of variations problem, where the infimum is taken over functions corresponding to the delta hedging terms and marginal constraint, and the supremum is taken over all market-calibrated models. The proof is given in Sects. 6.3 and 6.5.

Remark 3.7 Recall from Remark 2.3 that the case of strict inequality in (2.2) may be thought of as a natural model for a financial bubble. From (3.2), we see that this never happens when call options are traded, $\mathcal{X}=\mathcal{X}_{c}$. It is still possible that

$$
s_{0}>c_{n}(0)=P_{\mathcal{X}_{c}, \mathcal{P}, \mathfrak{P}}\left(\mathbb{S}_{n}\right)=V_{\mathcal{X}_{c}, \mathcal{P}, \mathfrak{P}}\left(\mathbb{S}_{n}\right)
$$

so that the market price for the asset $\mathbb{S}$, which is $s_{0}$, is strictly greater than its fundamental price $c_{n}(0)$. However, it is not clear if this could be seen as a bubble. In this case, the market does not satisfy the no dominance principle of Merton [33]: the asset $\mathbb{S}_{n}$ is strictly dominated by a call with zero strike. This situation is akin to the case of bubbles in complete markets described in Jarrow et al. [28]. We shall see in Sect. 4.2 below that bubbles appear in a meaningful way when put options and not call options are traded. 
Note that by Proposition 3.2, no WFLVR is equivalent to $\mathcal{M}_{\mathcal{X}}^{-}, \mathcal{P}, \mathfrak{P} \neq \emptyset$ and implies Condition 3.1. Following classical arguments going back to Breeden and Litzenberger [7], we can then define probability measures $\mu_{i}$ on $\mathbb{R}_{+}$by

$$
\mu_{i}([0, K])=1+c_{i}^{\prime}(K) \quad \text { for } K \in \mathbb{R}_{+} .
$$

Naturally the market prices $\mathcal{P}$, or $c_{i}(K)$, are uniquely encoded via $\mu_{i}$ with

$$
c_{i}(K)=\mathcal{P}\left(\left(\mathbb{S}_{i}-K\right)^{+}\right)=\int(s-K)^{+} \mu_{i}(\mathrm{~d} s) .
$$

To make the link with the (super-)martingale transport explicit, we may think of $\left(\mu_{i}\right)$ as the inputs. Note that by Remark 2.4, the set of market-calibrated models $\mathcal{M}_{\mathcal{X}_{c}, \mathcal{P}, \mathfrak{P}}$ is simply the set of probability measures $\mathbb{P}$ on $\mathbb{R}_{+}^{n}$ such that $\mathbb{S}_{0}=s_{0}$, $\mathbb{S}$ is a supermartingale and $\mathbb{S}_{i}$ is distributed according to the measure $\mu_{i}$. Accordingly we use the notation $\mathcal{M}_{\mathcal{X}_{c}, \mathcal{P}, \mathfrak{P}}^{-}=\mathcal{M}_{\mu, \mathfrak{P}}^{-}$and $P_{\mu, \mathfrak{P}}(G):=\sup _{\mathbb{P} \in \mathcal{M}_{\mu, \mathfrak{P}}^{-}} \mathbb{E}_{\mathbb{P}}[G]$. Likewise we write $V_{\mathcal{X}, \mathcal{P}, \mathfrak{P}}(G)=V_{\boldsymbol{\mu}, \mathfrak{P}}(G)$. Note that we have dropped the explicit reference to call options. This is justified since in fact we can allow any $\mu_{i}$-integrable functions for the static part of trading strategies. To state this as a corollary, we first rewrite Condition 3.1 in terms of $\mu_{1}, \ldots, \mu_{n}$ as follows:

Assumption 3.8 The probability measures $\mu_{1}, \ldots, \mu_{n}$ on $\mathbb{R}_{+}$satisfy

1. $s_{0} \geq \int_{\mathbb{R}_{+}} x \mu_{1}(\mathrm{~d} x) \geq \cdots \geq \int_{\mathbb{R}_{+}} x \mu_{n}(\mathrm{~d} x)$;

2. the sequence $\left(\int \phi \mathrm{d} \mu_{i}\right)_{1 \leq i \leq n}$ is nonincreasing for any concave and nondecreasing function $\phi: \mathbb{R} \rightarrow \mathbb{R}_{+}$.

Then Theorem 3.5 may be restated as follows.

Corollary 3.9 Assume $\mu_{1}, \ldots, \mu_{n}$ satisfy Assumption 3.8 and $\mathcal{M}_{\mu, \mathfrak{P}}^{-} \neq \emptyset$. Let the function $G: \mathbb{R}_{+}^{n} \rightarrow[-\infty, \infty)$ be upper semi-continuous and satisfy (3.1). Then

$$
\begin{gathered}
P_{\boldsymbol{\mu}, \mathfrak{P}}(G)=\inf \left\{\sum_{i=0}^{n} \int u_{i}(s) \mu_{i}(\mathrm{~d} s): u_{i}: \mathbb{R}_{+} \rightarrow \mathbb{R}\right. \text { with linear growth and } \\
\qquad \begin{array}{c}
\Delta_{i}: \mathbb{R}_{+}^{i} \rightarrow \mathbb{R}_{+} \text {bounded with } \\
\left.\Psi_{\left(u_{i}\right),\left(\Delta_{i}\right)} \geq G \text { on } \mathfrak{P}\right\} \\
=V_{\boldsymbol{\mu}, \mathfrak{P}}(G),
\end{array}
\end{gathered}
$$

where $\mu_{0}:=\delta_{s_{0}}$. Further, if $\int x \mu_{i}(\mathrm{~d} x)=s_{0}$ for $i=1, \ldots, n$, then (3.4) also holds with $\Delta_{i}: \mathbb{R}_{+}^{i} \rightarrow \mathbb{R}$. 
Proof By taking expectations in the pathwise superhedging inequality, we have for any $\mathbb{P} \in \mathcal{M}_{\boldsymbol{\mu}, \mathfrak{P}}^{-}$and any superhedging strategy $\left(\left(u_{i}\right),\left(\Delta_{i}\right)\right)$ as in (3.4) that

$$
\mathbb{E}_{\mathbb{P}}[G] \leq \sum_{i=1}^{n} \int u_{i}(s) \mu_{i}(\mathrm{~d} s),
$$

and hence the inequality " $\leq$ " in the first equality in (3.4) follows; see also Remark 2.3. The inequality " $\leq$ " in the second equality in (3.4) is obvious because we take the infimum over a smaller set of superhedging strategies. The result (3.4) then follows by Theorem 3.5. The last statement is clear since in the special case that $\mu_{1}, \ldots, \mu_{n}$ have the same mean, $\mathcal{M}_{\boldsymbol{\mu}, \mathfrak{P}}^{-}$is the set of martingale measures with marginals $\mu_{i}$.

Remark 3.10 Note that (3.4) is a generalisation of Corollary 1.1 in Beiglböck et al. [4] to a setup including a prediction set $\mathfrak{P}$ and with marginals having nonincreasing means.

The implication of Corollary 3.9 is that in our setup corresponding to a market without bubbles, a ban on short selling does not make any difference to the robust superhedging prices, and the martingale transport cost of $G$ with prediction set $\mathfrak{P}$ is equal to the robust (P)-superhedging price of $G$.

\section{Put options as hedging instruments}

We specify now to the case $\mathcal{X}=\mathcal{X}_{p}$ when put options are traded. The set of semistatic trading strategies $(X, \Delta)$ is denoted by $\mathcal{A}_{p}$. In this case, the options cannot be used to superreplicate the asset. This, as we shall see, has important consequences for pricing and hedging.

\subsection{Pricing-hedging duality for options with bounded payoffs}

We start with a brief discussion of the market input, no arbitrage, and existence of market-calibrated models.

Condition 4.1 Let $\mathcal{X}=\mathcal{X}_{p}$ and $p_{i}(K):=\mathcal{P}\left(\left(K-\mathbb{S}_{i}\right)^{+}\right), i=1, \ldots, n, K \geq 0$. Then

(i) $p_{i}(x)$ is a nonnegative, convex, increasing function of $x$ on $\mathbb{R}_{+}$;

(ii) $s_{0} \geq \lim _{x \rightarrow \infty}\left(x-p_{1}(x)\right) \geq \cdots \geq \lim _{x \rightarrow \infty}\left(x-p_{n}(x)\right) \geq 0$, and for every $i$, $0 \leq p_{i}^{\prime}(0+) \leq 1$

(iii) $p_{i}(K) \rightarrow 0$ as $K \rightarrow 0$;

(iv) for any $x \in \mathbb{R}_{+}, p_{i}(x)$ is nondecreasing in $i$.

A robust fundamental theorem of asset pricing analogous to the one in Proposition 3.2 holds also in this setup.

Proposition 4.2 Suppose $\mathfrak{P}$ is a closed subset of $\Omega$ and $\mathcal{X}=\mathcal{X}_{p}$. Then there is no WFLVR if and only if there exists a market-calibrated model, which then implies Condition 4.1. Furthermore, if $\mathfrak{P}=\Omega$, then Condition 4.1 implies the absence of WFLVR. 
A direct analogue of Proposition 3.3 holds also in this setup. Further, if Condition 4.1 is satisfied, similarly to (3.3), we can define probability measures $\mu_{i}$ on $\mathbb{R}_{+}$ by

$$
\mu_{i}([0, K])=p_{i}^{\prime}(K) \quad \text { for } K \in \mathbb{R}_{+}
$$

which satisfy the same properties as before, namely Assumption 3.8. The set of market-calibrated models is simply $\mathcal{M}_{\mathcal{X}_{p}, \mathcal{P}, \mathfrak{P}}^{-}=\mathcal{M}_{\boldsymbol{\mu}, \mathfrak{P}}^{-}$and only depends on the marginals $\mu_{i}$ and not on whether these were derived from put or from call prices. In consequence, we have $P_{\mathcal{X}_{p}, \mathcal{P}, \mathfrak{P}}(G)=P_{\mu, \mathfrak{P}}(G)$.

The situation on the dual side-the superhedging problem-is different. Indeed, we have seen in Corollary 3.9 that in the case of call options, we could relax the static part of the portfolio from combinations of call options to combinations of any functions with linear growth without affecting the superhedging price. In contrast, when put options are traded, their combinations are always bounded and such a relaxation is not possible. We stress this in the notation and write $V_{\mu, \mathfrak{P}}^{(p)}(G):=V_{\mathcal{X}}, \mathcal{P}, \mathfrak{P}(G)$. Our first result shows that when $G$ is bounded, then trading puts instead of calls has no impact on the superhedging price, as one would expect.

Theorem 4.3 Suppose the market input $\left(\mathcal{X}_{p}, \mathcal{P}, \mathfrak{P}\right)$ admits no WFLVR or equivalently that $\mathcal{M}_{\boldsymbol{\mu}, \mathfrak{P}}^{-} \neq \emptyset$. In particular, Condition 4.1 is satisfied and (4.1) defines measures which satisfy Assumption 3.8. Let $G: \mathbb{R}_{+}^{n} \rightarrow[-\infty, \infty)$ be an upper semicontinuous function bounded from above. Then

$$
P_{\mu, \mathfrak{P}}(G)=P_{\mathcal{X}_{p}, \mathcal{P}, \mathfrak{P}}(G)=V_{\mathcal{X}_{p}, \mathcal{P}, \mathfrak{P}}(G)=V_{\mu, \mathfrak{P}}^{(p)}(G)
$$

The proof is given in Sects. 6.3 and 6.5 and is similar to the proof of Theorem 3.5.

The above result may be extended to functions $G$ which are not necessarily bounded, but have sublinear growth. We state one such extension which is used later. In contrast, the duality in (4.2) will fail for $G$ which have linear growth - a theme we explore in the subsequent sections.

Corollary 4.4 In the setup of Theorem 4.3, assume that $G$ is an upper semicontinuous function such that $G_{M}\left(s_{1}, \ldots, s_{n}\right):=G\left(s_{1}, \ldots, s_{n}\right)-\left(\sum_{i=1}^{n} s_{i}\right) / M$ is bounded from above for any $M>1$. Then (4.2) holds for $G$.

Proof We have

$$
\begin{aligned}
V_{\boldsymbol{\mu}, \mathfrak{P}}^{(p)}(G) & \leq V_{\boldsymbol{\mu}, \mathfrak{P}}^{(p)}\left(G_{M}\right)+V_{\mu, \mathfrak{P}}^{(p)}\left(\left(\mathbb{S}_{1}+\cdots+\mathbb{S}_{n}\right) / M\right) \\
& \leq P_{\boldsymbol{\mu}, \mathfrak{P}}\left(G_{M}\right)+V_{\boldsymbol{\mu}, \mathfrak{P}}^{(p)}\left(\left(\mathbb{S}_{1}+\cdots+\mathbb{S}_{n}\right) / M\right) \\
& \leq P_{\boldsymbol{\mu}, \mathfrak{P}}(G)+\frac{n s_{0}}{M}
\end{aligned}
$$


where we used the obvious inequality $0 \leq V_{\mu, \mathfrak{P}}^{(p)}\left(\mathbb{S}_{i}\right) \leq s_{0}, i=1, \ldots, n$. By letting $M \rightarrow \infty$, we get $V_{\mu, \mathfrak{P}}^{(p)}(G) \leq P_{\mu, \mathfrak{P}}(G)$. The other inequality $V_{\mu, \mathfrak{P}}^{(p)}(G) \geq P_{\mu, \mathfrak{P}}(G)$ is true in full generality (see Remark 2.3), and we conclude that $V_{\mu, \mathfrak{P}}^{(p)}(G)=P_{\mu, \mathfrak{P}}(G)$.

\subsection{Duality gap and bubbles}

We come back to the topic of financial bubbles considered in Remarks 2.3 and 3.7. We start with a motivating example of a simple one-period model, $n=1$. The prediction set is of the form $\mathfrak{P}=\left\{s_{0}\right\} \times \mathfrak{P}_{1}$ for some $\mathfrak{P}_{1} \subset \mathbb{R}_{+}$. We assume the market admits no WFLVR, which is equivalent to saying that $\mu$ defined via (4.1) is a probability measure supported on $\mathfrak{P}_{1}$ and satisfies $\int x \mu(\mathrm{d} x) \leq s_{0}$. We assume the prediction set $\mathfrak{P}_{1}$ is unbounded and consider an option with an upper semi-continuous payoff function $G: \mathbb{R}_{+} \rightarrow[-\infty, \infty)$ such that $|G(x)| \leq K|x|$ for some $K$ and let

$$
\limsup _{x \rightarrow \infty, x \in \mathfrak{P}_{1}} \frac{G(x)}{x}=: \beta \in[-\infty, \infty) .
$$

A semi-static trading strategy $(X, \Delta) \in \mathcal{A}_{p}$ here is a pair with $X \in \mathcal{X}_{p}$ and $\Delta \geq 0$. If it superreplicates $G$, i.e.,

$$
\Psi_{X, \Delta}\left(s_{1}\right):=X\left(s_{1}\right)+\Delta\left(s_{1}-s_{0}\right) \geq G\left(s_{1}\right), \quad s_{1} \in \mathfrak{P}_{1},
$$

then necessarily $\Delta \geq \beta^{+}$since $X$ is bounded, where $\beta^{+}=\beta \vee 0$. Therefore, we find

$$
\begin{aligned}
V_{\mu, \mathfrak{P}}^{(p)}(G)= & \inf \left\{\int\left(s_{1}\right) \mu\left(\mathrm{d} s_{1}\right):(X, \Delta) \in \mathcal{A}_{p} \text { with } \Psi_{X, \Delta} \geq G \text { on } \mathfrak{P}\right\} \\
= & \inf _{\Delta_{0} \geq \beta^{+}}\left(\Delta_{0} s_{0}+\inf \left\{\int X\left(s_{1}\right) \mu\left(\mathrm{d} s_{1}\right):(X, \Delta) \in \mathcal{A}_{p}\right. \text { with }\right. \\
\left.\left.\qquad \Psi_{X, \Delta}\left(s_{1}\right) \geq G\left(s_{1}\right)-\Delta_{0} s_{1} \forall s_{1} \in \mathfrak{P}_{1}\right\}\right) & \\
= & \inf _{\Delta_{0} \geq \beta^{+}}\left(\Delta_{0} s_{0}+P_{\mu, \mathfrak{P}}\left(G(\mathbb{S})-\Delta_{0} \mathbb{S}_{1}\right)\right) \\
= & \inf _{\Delta_{0} \geq \beta^{+}}\left(\Delta_{0} s_{0}+\int_{\mathbb{R}_{+}}\left(G\left(s_{1}\right)-\Delta_{0} s_{1}\right) \mu\left(\mathrm{d} s_{1}\right)\right) \\
= & \int_{\mathbb{R}_{+}} G\left(s_{1}\right) \mu\left(\mathrm{d} s_{1}\right)+\inf _{\Delta_{0} \geq \beta^{+}}\left(\Delta_{0}\left(s_{0}-\int_{\mathbb{R}_{+}} s_{1} \mu\left(\mathrm{d} s_{1}\right)\right)\right) \\
= & \int_{\mathbb{R}_{+}} G\left(s_{1}\right) \mu\left(\mathrm{d} s_{1}\right)+\beta^{+}\left(s_{0}-\int_{\mathbb{R}_{+}} s_{1} \mu\left(\mathrm{d} s_{1}\right)\right) \\
= & P_{\mu, \mathfrak{P}}(G)+\beta^{+}\left(s_{0}-\int_{\mathbb{R}_{+}} s_{1} \mu\left(\mathrm{d} s_{1}\right)\right) .
\end{aligned}
$$

It follows that if the mean of $\mu$ is strictly smaller than $s_{0}$, then we have a duality gap for $G$ with linear growth. The intuitive reason is clear: buying the asset di- 
rectly is implicitly more expensive than constructing a position using put options. If $G$ has a bounded payoff, then the latter is feasible as seen in Theorem 4.3. However, for $G$ with a linear growth, any superhedging portfolio has to include the asset $\mathbb{S}$ and is hence more expensive, as seen above. When $G\left(s_{1}\right)=\left(s_{1}-K\right)^{+}$, $\lim \sup _{x \rightarrow \infty, x \in \mathfrak{P}_{1}} G(x) / x=1$ and we obtain

$$
V_{\mu, \mathfrak{P}}^{(p)}(G)=P_{\mu, \mathfrak{P}}(G)+\left(s_{0}-\int_{\mathbb{R}_{+}} x \mu(\mathrm{d} x)\right) .
$$

Likewise, taking $G\left(s_{1}\right)=s_{1}$, we have

$$
s_{0}=V_{\mu}^{(p)}\left(\mathbb{S}_{1}\right) \geq P_{\mu, \mathfrak{P}}\left(\mathbb{S}_{1}\right)=f_{0}:=\int_{\mathbb{R}_{+}} s_{1} \mu\left(\mathrm{d} s_{1}\right) .
$$

The market has a bubble - a misalignment of market and fundamental prices - if the forward price $f_{0}$ implied by the put options is strictly smaller than the spot price $s_{0}$. This should be contrasted with the situation in Remark 3.7, where the bubble arose due to dominated assets.

The difference between these situations can be summarised as follows: in order to have a financially meaningful market, we must always have the inequalities

$$
\begin{aligned}
& \text { market price } \geq \text { cheapest superreplication price } \\
& \geq \sup \{\text { model-implied prices }\} \\
&=\text { fundamental price. }
\end{aligned}
$$

The first inequality here follows from the fact that we can superreplicate an asset by purchasing it, and we may have a strict inequality without creating an arbitrage opportunity if it is not possible (due to portfolio constraints) to short sell the asset. However, in the case where there is a strict inequality here, the market contains a dominating portfolio - that is, the superreplicating strategy strictly dominates the purchase of the asset at the market price, and so Merton's no dominance principle fails. In general, one would not expect such markets to exist-even if arbitrage were not possible, one would expect equilibrium to close the gap, since no (rational) market participants would purchase the asset at its market price. On the other hand, the second inequality here is rational-there is no a priori need for the superreplication price and the model-implied price to agree.

As a result, markets where the fundamental price and the market price differ for some assets, but where the fundamental price and the superreplication price always agree, are mathematically possible, even if they are not economically plausible. This corresponds to the setup described in Sect. 3. A second case is also possible, and economically more plausible, where the superreplication price of an asset coincides with its market price but is different from the fundamental price. This corresponds to the setup described in Sect. 4. One of the main contributions of this paper is that we provide specific characterisations of markets where both behaviours are possible.

In a more classical framework, the two cases described above are encapsulated in the difference between the complete setting of Cox and Hobson [10] and Jarrow 
et al. [28], and the incomplete models of Jarrow et al. [29]. In the former, completeness of the market implies that equality always holds between the cost of the cheapest superreplicating strategy for an option and the model-implied price of the option. In contrast, in the latter, Merton's no-dominance condition implies that the inequality between the market price and the superreplication price is an equality. However, in Jarrow et al. [29], the existence of a bubble depends on the choice of some pricing measure to determine the "market-price". In the current (robust) setting, we are able to define the fundamental price in a concrete way (dependent only on the market prices and the prediction set), leading to a possibly clearer characterisation of a bubble, which does not need some external "selection" procedure.

We now extend the above discussion to a general $n$-marginal setting. In the oneperiod case above, any $G$ was a European option and the size of the gap between its market and fundamental prices was given simply as a product of its linear growth coefficient and the bubble size $s_{0}-f_{0}$. In the general setting, we cannot compute explicitly the duality gap for an arbitrary payoff $G$. We give below a characterisation which then allows us to obtain explicit expressions for most of the typically traded exotic options.

Theorem 4.5 Assume $\mu_{1}, \ldots, \mu_{n}$ satisfy Assumption 3.8. Suppose the payoff function $G: \mathbb{R}_{+}^{n} \rightarrow[-\infty, \infty)$ is upper semi-continuous and satisfies (3.1) on $\mathbb{R}_{+}^{n}$ for some $K$. Define $\beta_{i}: \mathbb{R}_{+}^{i} \rightarrow \mathbb{R}$ recursively by setting $\beta_{n}=0$ and

$$
\begin{aligned}
& \beta_{i}\left(s_{1}, \ldots, s_{i}\right) \\
& =\sup _{s_{i+2}, \ldots, s_{n} \in \mathbb{R}_{+}} \limsup _{x \rightarrow \infty}\left(\left(\beta_{i+1}\left(s_{1}, \ldots, s_{i}, x\right)\right.\right. \\
& \left.\left.\quad+\frac{G\left(s_{1}, \ldots, s_{i}, x, s_{i+2}, \ldots, s_{n}\right)}{x}\right) \mathbb{1}_{\mathfrak{P}}\left(s_{1}, \ldots, s_{i}, x, s_{i+2}, \ldots, s_{n}\right)\right) \vee 0,
\end{aligned}
$$

for $i=0, \ldots, n-1$. If $G_{\beta}(\mathbb{S}):=G(\mathbb{S})-\sum_{i=0}^{n-1} \beta_{i}\left(\mathbb{S}_{1}, \ldots, \mathbb{S}_{i}\right)\left(\mathbb{S}_{i+1}-\mathbb{S}_{i}\right)$ is upper semi-continuous and bounded from above, then

$$
V_{\boldsymbol{\mu}, \mathfrak{P}}^{(p)}(G)=\sup _{\mathbb{P} \in \mathcal{M}_{\boldsymbol{\mu}, \mathfrak{P}}^{-}} \mathbb{E}_{\mathbb{P}}\left[G-\sum_{i=0}^{n-1} \beta_{i}\left(\mathbb{S}_{1}, \ldots, \mathbb{S}_{i}\right)\left(\mathbb{S}_{i+1}-\mathbb{S}_{i}\right)\right]=P_{\boldsymbol{\mu}, \mathfrak{P}}\left(G_{\beta}\right) .
$$

More generally, the result remains true if there exists a sequence $\left(0, \beta^{(N)}\right) \in \mathcal{A}_{p}$ such that $G_{\beta^{(N)}}(\mathbb{S})$ is upper semi-continuous, bounded from above on $\mathfrak{P}$ for every $N$, and $G_{\beta^{(N)}}(\mathbb{S}) \rightarrow G_{\beta}(\mathbb{S})$ pointwise as $N \rightarrow \infty$.

The proof is reported in Sect. 6.6. Here we show how the above result applies in the case of an Asian or a lookback option when $\mathfrak{P}=\Omega$.

Remark 4.6 This result stands in stark contrast with the existing literature on pricing under constraints on short selling. For example, in a general (classical) setting, where prices are assumed to be locally bounded semimartingales under some probability 
measure $\mathbb{P}$, and under a restriction on short selling, Pulido [37, Theorem 4.1] shows that there is no duality gap.

Example 1 An Asian option has payoff function $G: \mathbb{R}_{+}^{n} \rightarrow \mathbb{R}_{+}$defined by

$$
G\left(s_{1}, \ldots, s_{n}\right)=\left(\frac{\sum_{i=1}^{n} s_{i}}{n}-K\right)^{+}
$$

In this case, as for any $i=1, \ldots, n$ and $s_{1}, \ldots, s_{i}, s_{i+2}, \ldots, s_{n} \in \mathbb{R}_{+}$, we have

$$
\lim _{x \rightarrow \infty} \frac{G\left(s_{1}, \ldots, s_{i}, x, s_{i+2}, \ldots, s_{n}\right)}{x}=\frac{1}{n},
$$

(4.4) can be simplified to

$$
\beta_{i}\left(s_{1}, \ldots, s_{i}\right)=\sup _{s_{i+2}, \ldots, s_{n} \in \mathbb{R}_{+}} \limsup _{x \rightarrow \infty} \beta_{i+1}\left(s_{1}, \ldots, s_{i}, x\right)+\frac{1}{n} .
$$

This yields $\beta_{i}=(n-i) / n$ for $i=0, \ldots, n-1$. It is clear that

$$
G_{\beta}(\mathbb{S})=G(\mathbb{S})-\sum_{i=0}^{n-1} \beta_{i}\left(\mathbb{S}_{i+1}-\mathbb{S}_{i}\right)=\left(\frac{\sum_{i=1}^{n} \mathbb{S}_{i}}{n}-K\right)^{+}-\frac{\sum_{i=1}^{n} \mathbb{S}_{i}}{n}+s_{0}
$$

is continuous and bounded from above. Therefore, by Theorem 4.5,

$$
\begin{aligned}
V_{\boldsymbol{\mu}}^{(p)}(G) & =\sup _{\mathbb{P} \in \mathcal{M}_{\mu}^{-}} \mathbb{E}_{\mathbb{P}}\left[G-\sum_{i=0}^{n-1} \beta_{i}\left(\mathbb{S}_{i+1}-\mathbb{S}_{i}\right)\right] \\
& =\sup _{\mathbb{P} \in \mathcal{M}_{\mu}^{-}} \mathbb{E}_{\mathbb{P}}[G]+\frac{1}{n} \sum_{i=1}^{n}\left(s_{0}-\int_{\mathbb{R}_{+}} x \mu_{i}(\mathrm{~d} x)\right) .
\end{aligned}
$$

Example 2 The second example we consider is a lookback option with a knock-in feature, whose payoff function $G: \mathbb{R}_{+}^{n} \rightarrow \mathbb{R}_{+}$is given by

$$
G\left(s_{1}, \ldots, s_{n}\right)=\left(\max _{0 \leq i \leq n} s_{i}-K\right)^{+} \mathbb{1}_{\left\{\min _{0 \leq i \leq n} s_{i} \leq B\right\}} .
$$

In particular, when $B=\infty$, it is just a lookback call option with strike $K$. By (4.4),

$$
\beta_{n-1}\left(s_{1}, \ldots, s_{n-1}\right)=\sup _{s_{n} \in \mathbb{R}_{+}} \limsup _{x \rightarrow \infty} \frac{G\left(s_{1}, \ldots, s_{n-1}, x\right)}{x}=\mathbb{1}_{\left\{\min _{0 \leq i \leq n-1} s_{i} \leq B\right\}} .
$$

Since for $i=0, \ldots, n-2$ and $s_{1}, \ldots, s_{i} \in \mathbb{R}_{+}$, we have

$$
\alpha_{i}\left(s_{1}, \ldots, s_{i}\right):=\sup _{s_{i+2}, \ldots, s_{n} \in \mathbb{R}_{+}} \lim _{x \rightarrow \infty} \frac{G\left(s_{1}, \ldots, s_{i}, x, s_{i+2}, \ldots, s_{n}\right)}{x}=1,
$$


(4.4) can then be simplified to

$$
\beta_{i}\left(s_{1}, \ldots, s_{i}\right)=\sup _{s_{i+2}, \ldots, s_{n} \in \mathbb{R}_{+}} \limsup _{x \rightarrow \infty} \beta_{i+1}\left(s_{1}, \ldots, s_{i}, x\right)+1, \quad i=0, \ldots, n-2,
$$

from which we can derive

$$
\beta_{i}\left(s_{1}, \ldots, s_{i}\right)=(n-1-i)+\mathbb{1}_{\left\{\min _{0 \leq j \leq i} s_{j} \leq B\right\}}, \quad i=0, \ldots, n-1 .
$$

It is not hard to see that

$$
\begin{aligned}
G_{\beta}(\mathbb{S})= & G(\mathbb{S})-\beta_{0}\left(\mathbb{S}_{1}-s_{0}\right)-\sum_{i=1}^{n-1} \beta_{i}\left(\mathbb{S}_{1}, \ldots, \mathbb{S}_{i}\right)\left(\mathbb{S}_{i+1}-\mathbb{S}_{i}\right) \\
= & G(\mathbb{S})+\beta_{0} s_{0}-\sum_{i=0}^{n-2}\left(\beta_{i}\left(\mathbb{S}_{1}, \ldots, \mathbb{S}_{i}\right)-\beta_{i+1}\left(\mathbb{S}_{1}, \ldots, \mathbb{S}_{i+1}\right)\right) \mathbb{S}_{i+1} \\
& -\beta_{n-1}\left(\mathbb{S}_{1}, \ldots, \mathbb{S}_{n-1}\right) \mathbb{S}_{n} \\
= & n s_{0}+\left(\max _{0 \leq i \leq n} \mathbb{S}_{i}-K\right)^{+} \mathbb{1}_{\left\{\min _{0 \leq i \leq n} \mathbb{S}_{i} \leq B\right\}}-\sum_{i=1}^{n} \mathbb{S}_{i} \\
& +\sum_{i=1}^{n} \mathbb{1}_{\left\{\min _{0 \leq j \leq i-1} \mathbb{S}_{j}>B\right\}} \mathbb{1}_{\left\{\mathbb{S}_{i} \leq B\right\}} \mathbb{S}_{i}
\end{aligned}
$$

is bounded from above. Now define continuous functions $\beta_{i}^{(N)}: \mathbb{R}_{+}^{i} \rightarrow \mathbb{R}_{+}$by

$$
\beta_{i}^{(N)}\left(s_{1}, \ldots, s_{i}\right)= \begin{cases}n-i & \text { if } \min _{0 \leq j \leq i} s_{j} \leq B, \\ n-i-1+N\left(B+\frac{1}{N}-\min _{0 \leq j \leq i} s_{j}\right)^{+} & \text {otherwise. }\end{cases}
$$

Similarly, we can show that $G_{\beta^{(N)}}(\mathbb{S})=G(\mathbb{S})-\sum_{i=0}^{n-1} \beta_{i}^{(N)}\left(\mathbb{S}_{1}, \ldots, \mathbb{S}_{i}\right)\left(\mathbb{S}_{i+1}-\mathbb{S}_{i}\right)$ is bounded from above. Also $\beta_{i}^{(N)} \rightarrow \beta_{i}$ as $N \rightarrow \infty$ for any $i=0, \ldots, n-1$. Then $G_{\beta^{(N)}} \rightarrow G_{\beta}$ pointwise as $N \rightarrow \infty$, and hence by Theorem 4.5,

$$
V_{\boldsymbol{\mu}}^{(p)}(G)=\sup _{\mathbb{P} \in \mathcal{M}_{\boldsymbol{\mu}}^{-}} \mathbb{E}_{\mathbb{P}}\left[G-\sum_{i=0}^{n-1} \beta_{i}\left(\mathbb{S}_{1}, \ldots, \mathbb{S}_{i}\right)\left(\mathbb{S}_{i+1}-\mathbb{S}_{i}\right)\right]
$$

As shown in the lookback option example above, the duality gap is not only dependent on $G$ and the marginal distributions $\mu_{i}$, but also on how the $\mu_{i}$ are (optimally) transported. In the case that $\mathfrak{P}$ is a strict subset of $\Omega$, it may become increasingly hard to calculate $\beta$ and check the assumption of Theorem 4.5. We develop now an argument which connects asymptotically the duality gap of $G$ in the presence of a prediction set and the duality gaps of penalised functions of $G$ in the absence of a prediction set. In particular, it provides an alternative way to compute the duality gap when $\mathfrak{P}$ is an arbitrary closed set. 
Assume the market input $\left(\mathcal{X}_{p}, \mathcal{P}, \mathfrak{P}\right)$ admits no WFLVR and $G$ is upper semicontinuous with

$$
G\left(s_{1}, \ldots, s_{n}\right) \leq K\left(1+s_{1}+\cdots+s_{n}\right), \quad\left(s_{1}, \ldots, s_{n}\right) \in \mathbb{R}_{+} .
$$

Under this assumption, we argue first that if $P_{\mu, \mathfrak{P}}(G)=-\infty$, then $V_{\mu, \mathfrak{P}}^{(p)}(G)=-\infty$. By Proposition 4.2, absence of WFLVR is equivalent to $\mathcal{M}_{\mathcal{X}_{p}, \mathcal{P}, \mathfrak{P}} \neq \emptyset$. It follows from the sublinearity of $V_{\boldsymbol{\mu}, \mathfrak{P}}^{(p)}$ and Theorem 4.3 that

$$
\begin{aligned}
V_{\mu, \mathfrak{P}}^{(p)}(G) & \leq V_{\mu, \mathfrak{P}}^{(p)}\left(G-K\left(1+\mathbb{S}_{1}+\cdots+\mathbb{S}_{n}\right)\right)+V_{\mu, \mathfrak{P}}^{(p)}\left(K\left(1+\mathbb{S}_{1}+\cdots+\mathbb{S}_{n}\right)\right) \\
& \leq P_{\boldsymbol{\mu}, \mathfrak{P}}\left(G-K\left(1+\mathbb{S}_{1}+\cdots+\mathbb{S}_{n}\right)\right)+n K s_{0}+K=-\infty
\end{aligned}
$$

From now on, we make the additional assumption that $P_{\mu, \mathfrak{P}}(G)>-\infty$. Define $G^{(N)}: \mathbb{R}_{+}^{n} \rightarrow[-\infty, \infty)$ by

$$
G^{(N)}\left(s_{1}, \ldots, s_{n}\right)=G\left(s_{1}, \ldots, s_{n}\right)-N \lambda_{\mathfrak{P}}\left(s_{1}, \ldots, s_{n}\right),
$$

where $\lambda_{\mathfrak{P}}(\mathbb{S}):=\left(1+\mathbb{S}_{1}+\cdots+\mathbb{S}_{n}\right) \mathbb{1}_{\left\{\left(\mathbb{S}_{1}, \ldots, \mathbb{S}_{n}\right) \notin \mathfrak{P}\right\}}$ is as in $(6.8)$. Then note that

$$
V_{\mu, \mathfrak{P}}^{(p)}(G)=\inf _{N \geq 1} V_{\mu}^{(p)}\left(G^{(N)}\right) .
$$

Indeed, the inequality " $\leq$ " is clear. On the other hand, " $\geq$ " follows from the fact that $G^{(N)}$ is decreasing in $N$, and given any $(X, \Delta) \in \mathcal{A}_{p}, \Psi_{X, \Delta} \geq-N\left(1+\sum_{i=1}^{N} \mathbb{S}_{i}\right)$ for $N$ sufficiently large.

Since $\mathfrak{P}$ is closed, $-\mathbb{1}_{\left\{\left(\mathbb{S}_{1}, \ldots, \mathbb{S}_{n}\right) \notin \mathfrak{P}\right\}}$ is an upper semi-continuous function and hence $G^{(N)}$ is upper semi-continuous. Then the problem is reduced to the case that $\mathfrak{P}=\Omega$, for which we have a formula to calculate the duality gap if the contingent claim satisfies all the assumptions in Theorem 4.5. Now let

$$
\gamma_{N}:=V_{\mu}^{(p)}\left(G^{(N)}\right)-\sup _{\mathbb{P} \in \mathcal{M}_{\mu}^{-}} \mathbb{E}_{\mathbb{P}}\left[G^{(N)}\right]
$$

It follows by (4.5) that

$$
V_{\boldsymbol{\mu}, \mathfrak{P}}^{(p)}(G)=\inf _{N \geq 1}\left(\sup _{\mathbb{P} \in \mathcal{M}_{\boldsymbol{\mu}}^{-}} \mathbb{E}_{\mathbb{P}}\left[G^{(N)}\right]+\gamma_{N}\right)=\lim _{N \rightarrow \infty}\left(\sup _{\mathbb{P} \in \mathcal{M}_{\boldsymbol{\mu}}^{-}} \mathbb{E}_{\mathbb{P}}\left[G^{(N)}\right]+\gamma_{N}\right) .
$$

In addition, we can deduce that

$$
\inf _{N \geq 1} \sup _{\mathbb{P} \in \mathcal{M}_{\mu}^{-}} \mathbb{E}_{\mathbb{P}}\left[G^{(N)}\right]=\sup _{\mathbb{P} \in \mathcal{M}_{\mu}^{-}} \inf _{N \geq 1} \mathbb{E}_{\mathbb{P}}\left[G^{(N)}\right]=\sup _{\mathbb{P} \in \mathcal{M}_{\mu, \mathfrak{P}}^{-}} \mathbb{E}_{\mathbb{P}}[G],
$$

where the first equality is achieved by using the min-max theorem [42, Corollary 2] and the second holds as $\inf _{N \geq 1} \mathbb{E}_{\mathbb{P}}\left[G-N \lambda_{\mathfrak{P}}\right]=-\infty$ for any $\mathbb{P} \in\left(\mathcal{M}_{\boldsymbol{\mu}}^{-} \backslash \mathcal{M}_{\mu, \mathfrak{P}}^{-}\right)$ but

$$
\inf _{N \geq 1} \sup _{\mathbb{P} \in \mathcal{M}_{\boldsymbol{\mu}}^{-}} \mathbb{E}_{\mathbb{P}}\left[G^{(N)}\right] \geq \sup _{\mathbb{P} \in \mathcal{M}_{\boldsymbol{\mu}, \mathfrak{P}}^{-}} \mathbb{E}_{\mathbb{P}}[G]>-\infty .
$$


Hence, the limit of $\left(\gamma_{N}\right)$ exists and by writing $\gamma=\lim _{N \rightarrow \infty} \gamma_{N}$, we have

$$
V_{\boldsymbol{\mu}, \mathfrak{P}}^{(p)}(G)=\sup _{\mathbb{P} \in \mathcal{M}_{\boldsymbol{\mu}, \mathfrak{P}}^{-}} \mathbb{E}_{\mathbb{P}}[G]+\gamma .
$$

\section{Continuous time: local martingales, bubbles and pricing}

We now turn to continuous-time models and explore the link between option prices, trading constraints, speculative bubbles and strict local martingales. Let $\Omega=\mathbb{D}\left([0, T], \mathbb{R}_{+}\right)$be the space of nonnegative right-continuous functions with left limits on $[0, T]$ and $\mathbb{S}=\left(\mathbb{S}_{t}: t \leq T\right)$ the canonical process on $\Omega$ with $\left(\mathcal{F}_{t}\right)$ denoting its natural filtration.

Now let us consider the case when put options are traded for $n \geq 1$ maturities $0<T_{1}<\cdots<T_{n}=T$,

$$
\mathcal{X}_{p}:=\left\{\left(K-\mathbb{S}_{T_{i}}\right)^{+}: 1 \leq i \leq n, K \geq 0\right\}, \quad p_{i}(K):=\mathcal{P}\left(\left(K-\mathbb{S}_{T_{i}}\right)^{+}\right) .
$$

We need to impose some assumptions on the prediction set $\mathfrak{P}$.

Assumption 5.1 The prediction set $\mathfrak{P} \subset \Omega$ satisfies $\omega(0)=s_{0}$ for every $\omega \in \mathfrak{P}$ and

for any $\omega \in \mathfrak{P}$ and any stopping time $\tau, \quad \omega^{\tau}=(\omega(t \wedge \tau(\omega)): t \leq T) \in \mathfrak{P}$.

Further the set $\mathfrak{P}_{T}:=\left\{\left(\omega_{0}, \omega_{T_{1}}, \ldots, \omega_{T_{n}}\right): \omega \in \mathfrak{P}\right\}$ is closed.

The first condition corresponds to $\mathfrak{P}$ being closed under stopping and will imply that any superhedging strategy in fact satisfies a collateral requirement; see Remark 5.2 below. The second point is technical and will enable us to compare the continuous-time setting to the discrete-time setting.

There are several possible choices for the class of admissible dynamic trading strategies. They typically lead to the same superhedging price, provided the admissible class is large enough, but to different sets of market-calibrated models. Here, to make the connection with the discrete-time setup clearer, we consider dynamic trading strategies $\Delta$ which are predictable piecewise constant processes with finitely many jumps. More precisely, we consider $\Delta:[0, T] \times \Omega \rightarrow \mathbb{R}_{+}$such that for any $\omega \in \Omega, \Delta(\omega):[0, T] \rightarrow \mathbb{R}_{+}$is a simple nonnegative function (piecewise constant with finitely many jumps) and for any $t \in[0, T]$ and for any $\omega_{1}, \omega_{2} \in \Omega$ such that $\omega_{1}(s)=\omega_{2}(s)$ for $s \in[0, t)$, we have $\Delta_{t}\left(\omega_{1}\right)=\Delta_{t}\left(\omega_{2}\right)$. We call such $\Delta$ admissible and write $\Delta \in \mathcal{A}$. Note that for $\Delta \in \mathcal{A}$, the stochastic integral $\int_{0}^{t} \Delta_{u-} \mathrm{d} \mathbb{S}_{u}$ is a sum and hence defined pathwise.

An admissible semi-static trading strategy is a pair $(X, \Delta)$ with a linear combination of put options $X(\omega)=a_{0}+\sum_{i=1}^{m} a_{i} X_{i}(\omega), m \geq 0, a_{i} \in \mathbb{R}, X_{i} \in \mathcal{X}_{p}$, and $\Delta \in \mathcal{A}$. Its payoff at time $T$ is given by

$$
\Psi_{X, \Delta}(\mathbb{S})=X(\mathbb{S})+\int_{0}^{T} \Delta_{u-} \mathrm{dS}_{u}
$$


We recall that the family of admissible semi-static trading strategies is denoted by $\mathcal{A}_{p}=\mathcal{A}_{\mathcal{X}_{p}}$ and that the superreplication price $V_{\mathcal{X}_{p}, \mathcal{P}, \mathfrak{P}}$ is given in Definition 2.1.

Remark 5.2 Note that because $\mathfrak{P}$ is closed under stopping (cf. Assumption 5.1), it follows that if $(X, \Delta) \in \mathcal{A}_{p}$ superhedges $G$ on $\mathfrak{P}$, then in fact

$$
\Psi_{X, \Delta}\left(\mathbb{S}^{t}\right)=X\left(\mathbb{S}^{t}\right)+\int_{0}^{t} \Delta_{u-} \mathrm{d} \mathbb{S}_{u} \geq G\left(\mathbb{S}^{t}\right), \quad t \leq T, \text { on } \mathfrak{P},
$$

where $\mathbb{S}^{t}=\left(\mathbb{S}_{u \wedge t}: u \leq T\right)$. In other words, $(X, \Delta)$ satisfies a collateral requirement. As we shall see below, this feature will contribute towards the emergence of bubbles.

Static trading arguments, as in the proof of Proposition 3.2, show that absence of WFLVR implies that $p_{i}(K)$ satisfy the properties listed in Condition 4.1, and hence we can use (4.1) to define probability measures $\boldsymbol{\mu}=\left(\mu_{i}\right)_{i=1}^{n}$ which satisfy Assumption 3.8. The set of market-calibrated models $\mathcal{M}_{\mathcal{X}, \mathcal{P}, \mathfrak{P}}^{-}$is given as in Definition 2.2. Note that Remark 2.3 is in force, with the convention $\infty-\infty=-\infty$. Finally, let $\mathcal{M}_{\mu, \mathfrak{P}}^{\text {loc }}$ denote the set of all calibrated local martingale measures on $\left(\Omega, \mathbb{F}_{T}\right)$, i.e., all $\mathbb{P}$ such that $\mathbb{S}$ is a $\mathbb{P}$-local martingale and $\mathbb{E}_{\mathbb{P}}\left[\left(K-\mathbb{S}_{T_{i}}\right)^{+}\right]=p_{i}(K), K \geq 0$, or equivalently $\mathbb{S}_{T_{i}} \sim \mu_{i}, 1 \leq i \leq n$. It is easy to see that $\mathcal{M}_{\mathcal{X}, \mathcal{P}, \mathfrak{P}}^{-}$is the set of measures $\mathbb{P}$ under which $\mathbb{S}_{T_{i}} \sim \mu$ and $\mathbb{S}$ is a $\mathbb{P}$-supermartingale and in particular $\mathcal{M}_{\mu, \mathfrak{P}}^{\text {loc }} \subset \mathcal{M}_{\mathcal{X}, \mathcal{P}, \mathfrak{P}}^{-}$.

Consider now a European option with payoff $G(\omega)=G\left(\omega_{T_{n}}\right)$, or more generally an upper semi-continuous $G(\omega)=G\left(\omega_{T_{1}}, \ldots, \omega_{T_{n}}\right)$. We can then compare the present setting to that of a discrete $n$-period model with traded put options at prices $\mathcal{P}$, where short selling is prohibited, and with a prediction set $\mathfrak{P}_{T}$, as considered in Sect. 4. Denote the corresponding primal and dual values by $P_{\mathcal{X}_{p}, \mathcal{P}, \mathfrak{P}_{\mathbf{T}}}^{d}$ and $V_{\mathcal{X}_{p}, \mathcal{P}, \mathfrak{P}_{\mathrm{T}}}^{d}$. Note that the discrete superhedging problem naturally embeds into the continuous-time one. A discrete-time trading strategy corresponds to a nonnegative $\left(\Delta_{t}\right)$ constant on every $\left[T_{i}, T_{i+1}\right)$ for $i=0, \ldots, n-1$, which is in $\mathcal{A}$. On the primal side, for any $\mathbb{P} \in \mathcal{M}_{\mu, \mathfrak{P}}^{\text {loc }}$, the vector $\left(\mathbb{S}_{0}, \mathbb{S}_{T_{1}}, \ldots, \mathbb{S}_{T_{n}}\right)$ is a market-calibrated discretetime model; so for $G$ as above, continuous-time calibrated models are embedded in discrete ones. In summary,

$$
P_{\mathcal{X}_{p}, \mathcal{P}, \mathfrak{P}}(G) \leq P_{\mathcal{X}_{p}, \mathcal{P}, \mathfrak{P}_{\mathbf{T}}}^{d}(G) \quad \text { and } \quad V_{\mathcal{X}_{p}, \mathcal{P}, \mathfrak{P}}(G) \leq V_{\mathcal{X}_{p}, \mathcal{P}, \mathfrak{P}_{\mathbf{T}}}^{d}(G)
$$

In some cases, we can establish an equality in the first inequality. For example, when

$$
\mathfrak{P}=\left\{\omega \in \Omega: \omega(0)=s_{0}\right\},
$$

then any discrete-time market model may be seen as a continuous-time one with the asset being constant on any $\left[T_{i}, T_{i+1}\right)$. We can then conclude that there is no duality gap in the continuous-time setting from Theorem 4.3 and Corollary 4.4 in discrete time.

However, our prime interest is in the case when the pricing-hedging duality fails. We can use the results of Sect. 4.2 to understand the case of European options. 
Proposition 5.3 Suppose that the prediction set $\mathfrak{P}$ satisfies Assumption 5.1, that the set $\mathfrak{P}_{T}:=\{\omega(T): \omega \in \mathfrak{P}\}$ is unbounded, and that market prices are such that $\mathcal{M}_{\mu, \mathfrak{P}}^{\text {loc }} \neq \emptyset$. Consider a continuous function $G: \mathbb{R}_{+} \rightarrow[-\infty, \infty)$ with linear growth (i.e., (3.1) holds) such that the limit $\beta:=\lim _{s \rightarrow \infty, s \in \mathfrak{P}_{T}} \frac{G(s)}{s}$ is well defined and nonnegative. Then

$$
\begin{aligned}
V_{\mathcal{X}_{p}, \mathcal{P}, \mathfrak{P}}(G) & =\sup _{\mathbb{P} \in \mathcal{M}_{\mu, \mathfrak{P}}^{\text {loc }}} \lim _{n \rightarrow \infty} \mathbb{E}_{\mathbb{P}}\left[G ^ { + } \left(\mathbb{S}_{\left.\left.T \wedge \tau_{n}^{\mathbb{P}}\right)-G^{-}\left(\mathbb{S}_{T}\right)\right]}\right.\right. \\
& =\int G(s) \mu_{n}(\mathrm{~d} s)+\beta\left(s_{0}-\int_{\mathbb{R}_{+}} s \mu_{n}(\mathrm{~d} s)\right),
\end{aligned}
$$

where we implicitly set $G(\omega)=G(\omega(T)),\left(\tau_{n}^{\mathbb{P}}\right)$ is a localising sequence for $\mathbb{S}$ under $\mathbb{P}$, and $G^{+}=G \vee 0, G^{-}=-(G \wedge 0)$.

We first give two remarks before proving the above result.

Remark 5.4 If the forward price implicit in the put options,

$$
f_{0}=\int s \mu_{n}(\mathrm{~d} s)=\lim _{K \rightarrow \infty}\left(K-p_{n}(K)\right),
$$

is cheaper than the spot, $s_{0}>f_{0}$, then the market has a bubble. The market price $s_{0}$ is strictly greater than the fundamental price, given by

$$
\sup _{\mathbb{P} \in \mathcal{M}_{\mu, \mathfrak{P}}^{\mathrm{loc}}} \mathbb{E}_{\mathbb{P}}\left[G\left(\mathbb{S}_{T}\right)\right]=\int G(s) \mu_{n}(\mathrm{~d} s) .
$$

The correction is equal to $\beta^{+}\left(s_{0}-f_{0}\right)$. This is the same correction as exhibited in Cox and Hobson [10, Theorem 5.2]; see also Sect. 6.1 in Jarrow et al. [29]. In Cox and Hobson [10], the bubble was driven by a collateral requirement and a strict local martingale property. While the former is a natural trading restriction, the latter appears artificial. In our robust framework, a bubble is triggered by trading restrictions and properties of market prices of options. The difference is that we take market prices as given and adopt a robust framework. A bubble arises when these prices are misaligned with the asset price, $s_{0}>f_{0}$, while an arbitrage does not arise because of the trading restrictions. In our setup, the trading restrictions take the form of a short selling ban and, as highlighted in Remark 5.2 above, a collateral requirement.

Remark 5.5 The assumption $\mathcal{M}_{\mu, \mathfrak{P}}^{\text {loc }} \neq \emptyset$ is an implicit assumption on $\mathfrak{P}$ and market prices. It is satisfied e.g. when $\mathfrak{P}$ is equal to all paths, or all continuous paths, which start in $s_{0}$, and put prices $p_{i}(K)$ satisfy the properties listed in Condition 4.1. The latter is equivalent to $\boldsymbol{\mu}=\left(\mu_{i}\right)_{i=1}^{n}$, defined via (4.1), satisfying Assumption 3.8.

Proof of Proposition 5.3 As explained above, we can directly compare the continuous-time setting with a discrete-time setting from Sect. 4 with the same put prices and prediction set

$$
\mathfrak{P}_{T}=\left\{\left(\omega_{0}, \omega_{T_{1}}, \ldots, \omega_{T_{n}}\right): \omega \in \mathfrak{P}\right\} .
$$


Using (4.3), which is a one-marginal result, we immediately have

$$
V_{\mathcal{X}_{p}, \mathcal{P}, \mathfrak{P}_{\mathbf{T}}}^{d}(G) \leq V_{\mu_{n}, \mathfrak{P}_{T}}^{p}(G)=\int G(s) \mu_{n}(\mathrm{~d} s)+\beta^{+}\left(s_{0}-\int_{\mathbb{R}_{+}} s \mu_{n}(\mathrm{~d} s)\right)
$$

and hence we conclude that

$$
V_{\mathcal{X}_{p}, \mathcal{P}, \mathfrak{P}}(G) \leq \int G(s) \mu_{n}(\mathrm{~d} s)+\beta^{+}\left(s_{0}-\int_{\mathbb{R}_{+}} s \mu_{n}(\mathrm{~d} s)\right) .
$$

Consider a superhedging strategy $(X, \Delta)$ and $\mathbb{P} \in \mathcal{M}_{\boldsymbol{\mu}, \mathfrak{P}}^{\text {loc }}$ with a reducing sequence $\left(\tau_{n}\right)$ for $\mathbb{S}$ under $\mathbb{P}$. If $G\left(\mathbb{S}_{T \wedge \tau_{n}}\right)>0$, it follows from $(5.1)$ and $G^{-} \geq 0$ that

$$
X\left(\mathbb{S}_{T \wedge \tau_{n}}\right)+\int_{0}^{T \wedge \tau_{n}} \Delta_{u-} \mathrm{d} \mathbb{S}_{u} \geq G^{+}\left(\mathbb{S}_{T \wedge \tau_{n}}\right)-G^{-}\left(\mathbb{S}_{T}\right) .
$$

Otherwise, $G\left(\mathbb{S}_{T \wedge \tau_{n}}\right) \leq 0$ and then

$$
X\left(\mathbb{S}_{T}\right)+\int_{0}^{T} \Delta_{u-} \mathrm{d} \mathbb{S}_{u} \geq G\left(\mathbb{S}_{T}\right) \geq G^{+}\left(\mathbb{S}_{T \wedge \tau_{n}}\right)-G^{-}\left(\mathbb{S}_{T}\right)
$$

Therefore,

$$
\begin{aligned}
G^{+}\left(\mathbb{S}_{T \wedge \tau_{n}}\right)-G^{-}\left(\mathbb{S}_{T}\right) \leq & X\left(\mathbb{S}_{T}\right)+\left(X\left(\mathbb{S}_{T \wedge \tau_{n}}\right)-X\left(\mathbb{S}_{T}\right)\right) \mathbb{1}_{\left\{G\left(\mathbb{S}_{T \wedge \tau_{n}}\right)>0\right\}} \\
& +\int_{0}^{T} \tilde{\Delta}_{u-} \mathrm{d} \mathbb{S}_{u},
\end{aligned}
$$

where $\tilde{\Delta}_{u}=\Delta_{u} \mathbb{1}_{\left\{u \leq \tau_{n} \wedge T\right\}}+\Delta_{u} \mathbb{1}_{\left\{u>\tau_{n} \wedge T\right\}} \mathbb{1}_{\left\{G\left(\mathbb{S}_{T \wedge \tau_{n}}\right) \leq 0\right\}}$. We note that $\tilde{\Delta} \in \mathcal{A}$ and hence the expectation of the integral is nonpositive under $\mathbb{P}$. Further, $\tau_{n} \wedge T=T$ for $n$ large enough (which may depend on the path) and $X$ is bounded so that we may apply the dominated convergence theorem to conclude that

$$
\underset{n \rightarrow \infty}{\limsup } \mathbb{E}_{\mathbb{P}}\left[G^{+}\left(\mathbb{S}_{T \wedge \tau_{n}}\right)-G^{-}\left(\mathbb{S}_{T}\right)\right] \leq \mathbb{E}_{\mathbb{P}}\left[X\left(\mathbb{S}_{T}\right)\right]=\mathcal{P}(X)
$$

and hence the LHS in (5.3) is a lower bound on $V_{\mathcal{X}}, \mathcal{P}, \mathfrak{P}(G)$. Finally, we compute this lower bound and compare it with (5.2). Note that for any $\epsilon>0, G(s)-(\beta-\epsilon) s$ is bounded from below on $\mathfrak{P}_{T}$. It follows, applying Fatou's lemma and noting that $\mathbb{P} \in \mathcal{M}_{\boldsymbol{\mu}, \mathfrak{P}}^{\text {loc }}$ implies that $\mathbb{S}_{T}$ and $\mathbb{S}_{T \wedge \tau_{n}}$ are almost surely in $\mathfrak{P}_{T}$, that

$$
\begin{aligned}
\liminf _{n \rightarrow \infty} \mathbb{E}_{\mathbb{P}}\left[G^{+}\left(\mathbb{S}_{T \wedge \tau_{n}}\right)\right] & \geq \liminf _{n \rightarrow \infty} \mathbb{E}_{\mathbb{P}}\left[G^{+}\left(\mathbb{S}_{T \wedge \tau_{n}}\right)-(\beta-\epsilon) \mathbb{S}_{T \wedge \tau_{n}}\right]+(\beta-\epsilon) s_{0} \\
& \geq \mathbb{E}_{\mathbb{P}}\left[G^{+}\left(\mathbb{S}_{T}\right)-(\beta-\epsilon) \mathbb{S}_{T}\right]+(\beta-\epsilon) s_{0} \\
& =\int G^{+}(s) \mu_{n}(\mathrm{~d} s)+(\beta-\epsilon)\left(s_{0}-\int s \mu_{n}(\mathrm{~d} s)\right) .
\end{aligned}
$$

We conclude that the upper bound in (5.2) coincides with the lower bound obtained by taking $\epsilon \searrow 0$ and the infimum over superhedging strategies in (5.3), as required. 
The above statement may be extended to $G$ which depend on the values of the asset at the intermediate maturities, i.e. $G(\mathbb{S})=G\left(\mathbb{S}_{T_{1}}, \ldots, \mathbb{S}_{T_{n}}\right)$, by using Theorem 4.5. We do not pursue this here.

Acknowledgements Zhaoxu Hou gratefully acknowledges the support of the Oxford-Man Institute of Quantitative Finance and Balliol College in Oxford. Jan Obłój gratefully acknowledges funding received from the European Research Council under the European Union's Seventh Framework Programme (FP7/2007-2013)/ERC grant agreement No. 335421. Jan Obłój is also thankful to the Oxford-Man Institute of Quantitative Finance and St John's College in Oxford for their financial support. The authors thank Philip Protter and two anonymous referees for their very helpful comments on an earlier version of this paper.

Open Access This article is distributed under the terms of the Creative Commons Attribution 4.0 International License (http://creativecommons.org/licenses/by/4.0/), which permits unrestricted use, distribution, and reproduction in any medium, provided you give appropriate credit to the original author(s) and the source, provide a link to the Creative Commons license, and indicate if changes were made.

\section{Appendix}

\subsection{Preliminary results}

In this section and in Sects. 6.3 and 6.5, we assume that $\mu_{1}, \ldots, \mu_{n}$ are probability measures on $\mathbb{R}_{+}$which have a finite first moment. Let $\Pi_{\mu}$ be the set of all Borel probability measures on $\Omega$ with marginals $\delta_{s_{0}}, \mu_{1}, \ldots, \mu_{n}$ and denote by $\mathcal{M}_{\mu}^{-}$the set of probability measures $\mathbb{P}$ on $\Omega$ such that $\mathbb{S}$ is a supermartingale and $\mathbb{S}_{i}$ is distributed according to $\mu_{i}$. We also write $C_{b}\left(\mathbb{R}_{+}^{j}, \mathbb{R}_{+}\right)$to denote the set of continuous, bounded and nonnegative functions $f$ on $\mathbb{R}_{+}^{j}$ and $C_{c}\left(\mathbb{R}_{+}^{j}, \mathbb{R}_{+}\right)$for the subset of continuous, nonnegative and compactly supported functions.

Lemma 6.1 Let $\pi \in \Pi_{\mu}$. Then the following are equivalent:

1. $\pi \in \mathcal{M}_{\bar{\mu}}^{-}$.

2. For $0 \leq j \leq n-1$ and for every $\Delta \in C_{c}\left(\mathbb{R}_{+}^{j}, \mathbb{R}_{+}\right)$, we have

$$
\int_{\Omega} \Delta\left(x_{1}, \ldots, x_{j}\right)\left(x_{j+1}-x_{j}\right) \mathrm{d} \pi\left(x_{1}, \ldots, x_{n}\right) \leq 0 .
$$

Proof Claim 1 asserts that whenever $A \subseteq \mathbb{R}_{+}^{j}, j \leq n-1$, is Borel-measurable, then

$$
\int_{\Omega_{s_{0}}} \mathbb{1}_{A}\left(x_{1}, \ldots, x_{j}\right)\left(x_{j+1}-x_{j}\right) \mathrm{d} \pi\left(x_{1}, \ldots, x_{n}\right) \leq 0 .
$$

To see $(6.2) \Rightarrow(6.1)$, we fix any $j=0, \ldots, n-1$ and $\Delta \in C_{c}\left(\mathbb{R}_{+}^{j}, \mathbb{R}_{+}\right)$and define simple functions $f_{k}: \mathbb{R}_{+}^{j} \rightarrow \mathbb{R}$ by $f_{k}=2^{-k}\left\lfloor 2^{k} \Delta\right\rfloor$. Then $0 \leq f_{k} \uparrow \Delta$ and it follows from the dominated convergence theorem and (6.2) that (6.1) is satisfied.

To show $(6.1) \Rightarrow(6.2)$, first consider $A \subseteq \mathbb{R}_{+}^{j}$ such that $A$ is open and bounded. Since $\mathbb{1}_{A}$ is lower semi-continuous, there exists a sequence $\left(f_{k}\right)_{k \geq 1} \subseteq C_{c}\left(\mathbb{R}_{+}^{j}, \mathbb{R}_{+}\right)$ 
such that $0 \leq f_{k} \leq \mathbb{1}_{A}$ and $f_{k} \uparrow \mathbb{1}_{A}$. Therefore, the dominated convergence theorem implies that

$$
\int_{\Omega} \mathbb{1}_{A}\left(x_{1}, \ldots, x_{j}\right)\left(x_{j+1}-x_{j}\right) \mathrm{d} \pi\left(x_{1}, \ldots, x_{n}\right) \leq 0
$$

Now consider an arbitrary open set $A \subseteq \mathbb{R}_{+}^{j}$. We can write $A=\bigcup_{n \geq 1} A^{(n)}$ with $A^{(n)}:=A \cap\left\{S \in \mathbb{R}_{+}^{j}:\|S\|<n\right\}$ being open and bounded. Then by the dominated convergence theorem,

$$
\int_{\Omega} \mathbb{1}_{A}\left(x_{1}, \ldots, x_{j}\right)\left(x_{j+1}-x_{j}\right) \mathrm{d} \pi\left(x_{1}, \ldots, x_{n}\right) \leq 0 .
$$

Finally, if $A \subseteq \mathbb{R}_{+}^{j}$ is a Borel set, then by Corollary 3.12 in Bruckner et al. [8], for every $N>0$, there is an open set $A_{N} \subseteq \mathbb{R}_{+}^{j}$ such that $A \subseteq A_{N}$ with $\pi\left(A_{N}\right) \leq \pi(A)+\frac{1}{N}$. It follows that

$$
\begin{aligned}
\int_{\Omega} & \mathbb{1}_{A}\left(x_{j+1}-x_{j}\right) \mathrm{d} \pi\left(x_{1}, \ldots, x_{n}\right) \\
= & \int_{\Omega} \mathbb{1}_{A_{N}}\left(x_{j+1}-x_{j}\right) \mathrm{d} \pi\left(x_{1}, \ldots, x_{n}\right)-\int_{\Omega} \mathbb{1}_{A_{N} \backslash A}\left(x_{j+1}-x_{j}\right) \mathrm{d} \pi\left(x_{1}, \ldots, x_{n}\right) \\
\leq & \int_{\Omega} \mathbb{1}_{A_{N} \backslash A}\left(x_{1}, \ldots, x_{j}\right) x_{j} \mathrm{~d} \pi\left(x_{1}, \ldots, x_{n}\right) \\
= & \int_{A_{N} \backslash A} \mathbb{1}_{\left\{x_{j} \geq \sqrt{N}\right\}} x_{j} \mathrm{~d} \pi\left(x_{1}, \ldots, x_{n}\right)+\int_{A_{N} \backslash A} \mathbb{1}_{\left\{x_{j}<\sqrt{N}\right\}} x_{j} \mathrm{~d} \pi\left(x_{1}, \ldots, x_{n}\right) \\
\leq & \int_{\mathbb{R}_{+}} \mathbb{1}_{\left\{x_{j} \geq \sqrt{N}\right\}} x_{j} \mu_{j}(\mathrm{~d} x)+\frac{\sqrt{N}}{N} \longrightarrow 0 \quad \text { as } N \rightarrow \infty .
\end{aligned}
$$

Lemma 6.2 For a closed $\mathfrak{P} \subseteq \Omega$, the set $\mathcal{M}_{\mu, \mathfrak{P}}^{-}$is compact in the weak topology.

Proof Since $\mathcal{M}_{\boldsymbol{\mu}, \mathfrak{P}}^{-}$is a subset of the compact set $\Pi_{\mu}$, it suffices to prove that $\mathcal{M}_{\boldsymbol{\mu}, \mathfrak{P}}^{-}$ is a closed subset of $\Pi_{\mu}$. By Lemma 6.1,

$$
\mathcal{M}_{\mu}^{-}=\bigcap_{j=0}^{n-1} \bigcap_{\Delta \in C_{b}\left(\mathbb{R}_{+}^{j}, \mathbb{R}_{+}\right)}\left\{\int_{\mathbb{R}_{+}^{n}} \Delta\left(x_{1}, \ldots, x_{j}\right)\left(x_{j+1}-x_{j}\right) \mathrm{d} \pi\left(x_{1}, \ldots, x_{n}\right) \leq 0\right\} .
$$

Therefore, by Lemma 2.2 in Beiglböck et al. [4], $\mathcal{M}_{\mu}^{-}$is a closed subset of $\Pi_{\boldsymbol{\mu}}$ in the weak topology.

To show $\mathcal{M}_{\mu, \mathfrak{P}}^{-}$is a closed subset of $\mathcal{M}_{\mu}^{-}$, we take any sequence $\left(\mathbb{Q}_{n}\right)$ in $\mathcal{M}_{\mu}$ such that $\mathbb{Q}_{n}[\mathfrak{P}]=1$ and $\mathbb{Q}_{n} \rightarrow \mathbb{Q}$ for some $\mathbb{Q} \in \mathcal{M}_{\mu}$ as $n \rightarrow \infty$. Then by weak convergence of measures, for $\mathfrak{P} \subseteq \Omega$ closed, $\mathbb{Q}[\mathfrak{P}] \geq \lim \sup _{n \rightarrow \infty} \mathbb{Q}_{n}[\mathfrak{P}]=1$. It follows that $\mathcal{M}_{\mu, \mathfrak{P}}$ is a closed subset of $\mathcal{M}_{\mu}$ and hence closed in $\Pi_{\boldsymbol{\mu}}$ in the weak topology. 
To prove Theorems 3.5 and 4.3, we use the following version of the MongeKantorovich duality theorem, which is essentially Proposition 2.1 in Beiglböck et al. [4]. The proposition is rewritten here to suit the notation and purpose of this paper.

Lemma 6.3 For any $G$ that is upper semi-continuous and bounded from above, we have

$$
\sup _{\pi \in \Pi_{\mu}} \mathbb{E}_{\pi}[G]=\inf \left\{\mathcal{P}(X): X \in \mathcal{A}_{o} \text { with } X \geq G \text { on } \Omega\right\}, \quad \text { where } o \in\{c, p\} .
$$

Further, the result remains true with $o=c$ for any upper semi-continuous $G$ that satisfies Condition 3.1.

The call option case is just Proposition 2.1 in Beiglböck et al. [4]. The put option case follows from (A.1) in the proof of Proposition 2.1 in Beiglböck et al. [4], which in our notation simply states that for any $G$ that is upper semi-continuous and bounded from above,

$$
\begin{aligned}
\sup _{\pi \in \Pi_{\mu}} \mathbb{E}_{\pi}[G]=\inf \left\{\sum_{i=0}^{n} \int u_{i} \mathrm{~d} \mu_{i}:\right. & u_{i} \in C_{b}\left(\mathbb{R}_{+}, \mathbb{R}\right) \text { with } \\
& \left.\sum_{i=1}^{n} u_{i}\left(\mathbb{S}_{i}\right) \geq G(\mathbb{S}) \text { on } \Omega\right\} .
\end{aligned}
$$

Note that given any $f \in C_{b}\left(\mathbb{R}_{+}, \mathbb{R}\right), \epsilon>0$ and $i=1, \ldots, n$, there is some $u: \mathbb{R}_{+} \rightarrow \mathbb{R}$ of the form $a_{0}+\sum_{j=1}^{m} a_{j}\left(K_{j}-s_{j}\right)^{+}$with $u \geq f$ and $\int(u-f) \mathrm{d} \mu_{i}<\epsilon$. Therefore, we may change the class of admissible functions in (6.3) from $C_{b}\left(\mathbb{R}_{+}, \mathbb{R}\right)$ to $u: \mathbb{R}_{+} \rightarrow \mathbb{R}$ taking the form $a_{0}+\sum_{j=1}^{m} a_{j}\left(K_{j}-s\right)^{+}$.

\subsection{Proofs of the FTAP in the setting with traded calls or puts: case where $\mathfrak{P}=\Omega$}

The proofs of Propositions 3.2 and 4.2 are virtually identical; so we only give the proof of Proposition 3.2. We include with it the proof of Proposition 3.3. In this section, we only give the proof in the case where $\mathfrak{P}=\Omega$. This allows us then to prove Theorem 3.5 when $\mathfrak{P}=\Omega$ which in turn is used to establish the results when $\mathfrak{P} \subsetneq \Omega$.

Step 1. " $\exists$ MCM (market-calibrated model) $\Longrightarrow$ no WFLVR".

First we show that the existence of a market-calibrated model implies no WFLVR. Fix a market-calibrated model $\mathbb{P}$ and any $\left(X_{k}, \Delta_{k}\right) \in \mathcal{A}_{\mathcal{X}}$ and $(X, \Delta) \in \mathcal{A}_{\mathcal{X}}$ such that $\Psi_{X_{k}, \Delta_{k}} \rightarrow 0$ pointwise on $\mathfrak{P}, \lim _{k} \mathcal{P}\left(X_{k}\right)$ is well defined and $\Psi_{X_{k}, \Delta_{k}} \geq \Psi_{X, \Delta}$. Then by Fatou's lemma, we get

$$
\liminf _{k} \mathbb{E}_{\mathbb{P}}\left[\Psi_{X_{k}, \Delta_{k}}\right] \geq \mathbb{E}_{\mathbb{P}}\left[\liminf _{k} \Psi_{X_{k}, \Delta_{k}}\right]=0
$$

and hence

$$
\lim _{k} \mathcal{P}\left(X_{k}\right)=\lim _{k} \mathbb{E}_{\mathbb{P}}\left[X_{k}\right] \geq \liminf _{k} \mathbb{E}_{\mathbb{P}}\left[\Psi_{X_{k}, \Delta_{k}}\right] \geq \mathbb{E}_{\mathbb{P}}\left[\liminf _{k} \Psi_{X_{k}, \Delta_{k}}\right]=0 .
$$


Step 2. "no WFLVR $\Longrightarrow$ Condition 3.1".

It is straightforward and classical that the absence of a robust uniformly strong arbitrage implies Condition 3.1(i) and (ii). Note that since the $c_{i}(\cdot)$ are convex, $c_{i}(0+)$ is well defined. Let $\alpha_{i}:=\lim _{K \rightarrow \infty} c_{i}(K)$ which is well defined by Condition 3.1(i) with $\alpha_{i} \geq 0$ for any $i=1, \ldots, n$. If $\alpha_{i}>0$ for some $i=1, \ldots, n$, then $\left(X_{k},(0)\right)$ with $X_{k}=-\left(\mathbb{S}_{i}-k\right)^{+}$is a WFLVR since $X_{k} \rightarrow 0$ pointwise as $k \rightarrow \infty$ and $\mathcal{P}\left(X_{k}\right)=-c_{i}(k) \rightarrow-\alpha_{i}<0$. If Condition 3.1(iv) is violated, then for some $K \in \mathbb{R}_{+}$ and $i, c_{i}(0)-c_{i}(K)<c_{i+1}(0)-c_{i+1}(K)$. Consider

$$
\begin{aligned}
X & =\left(\mathbb{S}_{i}-0\right)^{+}-\left(\mathbb{S}_{i}-K\right)^{+}-\left(\mathbb{S}_{i+1}-0\right)^{+}+\left(\mathbb{S}_{i+1}-K\right)^{+} \in \mathcal{X}_{c}, \\
\Delta_{i} & =\mathbb{1}_{\left\{\mathbb{S}_{i}<K\right\}} \quad \text { and } \quad \Delta_{j}=0 \text { for } j \neq i .
\end{aligned}
$$

Then $(X, \Delta)$ is a robust uniformly strong arbitrage since $\Psi_{X, \Delta} \geq 0$, but $\mathcal{P}(X)<0$. We conclude that no WFLVR implies Condition 3.1. Moreover, absence of a robust uniformly strong arbitrage implies Condition 3.1(i), (ii) and (iv).

Step 3. "If $\mathfrak{P}=\Omega$ then Condition $3.1 \Longrightarrow \exists \mathrm{MCM}$ ".

Next we show that Condition 3.1 implies the existence of a market-calibrated model when $\mathfrak{P}=\Omega$. It follows from Condition 3.1(i)-(iii) that we can derive from the observed prices of call options probability measures $\boldsymbol{\mu}=\left(\mu_{1}, \ldots, \mu_{n}\right)$ with $\mu_{i}$ on $\left(\mathbb{R}_{+}, \mathcal{B}\left(\mathbb{R}_{+}\right)\right)$such that for any $i=1, \ldots, n$ and $K \in \mathbb{R}_{+}$,

$$
c_{i}(K):=\int(x-K)^{+} \mu_{i}(\mathrm{~d} x) \text { and } c_{i}(0)=\int x \mu_{i}(\mathrm{~d} x),
$$

where $\mathcal{B}\left(\mathbb{R}_{+}\right)$is the Borel $\sigma$-algebra of $\mathbb{R}_{+}$. In fact, due to Breeden and Litzenberger [7], $\mu_{i}$ can be defined via

$$
\mu_{i}([0, K])=1+c_{i}^{\prime}(K) \quad \text { for } K \in \mathbb{R}_{+} .
$$

In addition, given $\mu_{1}, \ldots, \mu_{n}$ derived from the observed market prices of call options, Strassen's theorem [41] states that Condition 3.1(iv) holds if and only if for any convex nonincreasing function $\phi: \mathbb{R}_{+} \rightarrow \mathbb{R}$, the sequence $\left(\int \phi d \mu_{i}\right)_{i \geq 1}$ is nondecreasing, which is the necessary and sufficient condition for the existence of a nonnegative supermartingale having marginals $\mu_{1}, \ldots, \mu_{n}$. Therefore, when $\mathfrak{P}=\Omega$, the absence of WFLVR implies the existence of a market-calibrated model which happens if and only if Condition 3.1 is satisfied.

Step 4. "Additional arguments for Proposition 3.3".

Given the above steps, to show Proposition 3.3, it remains to argue that Condition 3.1(i), (ii) and (iv) imply that there is no robust uniformly strong arbitrage when $\mathfrak{P}=\Omega$. Suppose to the contrary that there exists a semi-static strategy $(X, \Delta)$ such that $\Psi_{X, \Delta} \geq 0$ and $\mathcal{P}(X)<\epsilon<0$. As $X$ is a finite linear combination of elements of $\mathcal{X}_{c}$, we let $K_{\max }$ be the largest among the strikes of call options present in $X$. Then, for any $\delta>0$ small enough, there exists a sequence of functions $\left(c_{i}^{(\delta)}\right)_{i=1}^{n}$ satisfying Condition 3.1(i)-(iv) and such that $\left|c_{i}(K)-c_{i}^{(\delta)}(K)\right| \leq \delta$, for any $i=1, \ldots, n$ and $K \leq K_{\max }$. In fact, we can construct functions $\left(c_{i}^{(\delta)}\right)_{i=1}^{n}$ in the following way. For any $i=1, \ldots, n$, we can first define a function $\tilde{c}_{i}$ by $\tilde{c}_{i}(K)=c_{i}(0)-\left(1-\frac{\delta i}{2 n s_{0}}\right)\left(c_{i}(0)-c_{i}(K)\right)$ if $c_{i}^{\prime}(0+)<0$, and 
$\tilde{c}_{i}(K)=\left(c_{i}(0)-\frac{\delta(n+1-i)}{2 n K_{\max }} K\right) \vee 0$ otherwise. Note that if $c_{i}^{\prime}(0+)=0$, then $c_{j} \equiv c_{j}(0)$ for any $j \geq i$. Then $\left|\tilde{c}_{i}(K)-c_{i}(K)\right| \leq \delta / 2$ for $K \leq K_{\max }$, and for $\delta$ sufficiently small, $\left(\tilde{c}_{i}\right)_{i=1}^{n}$ satisfies Condition 3.1(i)-(iv) and $\tilde{c}_{i}(K)-\tilde{c}_{i}(0)$ is strictly decreasing in $i$ for $K \in\left(0, K_{\max }\right]$. Then, for any $i \leq n$, we can find a convex, decreasing function $c_{i}^{(\delta)}$ which approximates $\tilde{c}_{i}$ arbitrarily closely on $\left[0, K_{\max }\right]$ and satisfies $c_{i}^{(\delta)}(0)=\tilde{c}_{i}(0)$, $\tilde{c}_{i+1}(0)-\tilde{c}_{i+1} \geq c_{i}^{(\delta)}(0)-c_{i}^{(\delta)} \geq \tilde{c}_{i}(0)-\tilde{c}_{i}$ and $c_{i}^{(\delta)}(K) \rightarrow 0$ as $K \rightarrow \infty$. By the arguments above, with $\mathcal{P}^{(\delta)}$ corresponding to prices $\left(c_{i}^{(\delta)}\right), \mathcal{P}^{(\delta)}$ and $\left(c_{i}^{(\delta)}\right)$ satisfy no WFLVR and hence there is no robust uniformly strong arbitrage, so $\mathcal{P}^{(\delta)}(X) \geq 0$. However, we can take $\delta$ small enough so that $\left|\mathcal{P}(X)-\mathcal{P}^{(\delta)}(X)\right|<\epsilon / 2$ which gives the desired contradiction and completes the proof of Proposition 3.3.

\subsection{Proof of Theorems 3.5 and 4.3: case where $\mathfrak{P}=\Omega$ and $G$ is bounded}

We now give the proof of Theorems 3.5 and 4.3 for bounded and upper semicontinuous $G$ in the case where $\mathfrak{P}=\Omega$. In this case, we can apply Proposition 3.2 or 4.2. The proof of the general case will be postponed. Since the proof of Theorem 4.3 is virtually identical to that of Theorem 3.5, we only give the proof of Theorem 3.5.

Proof of Theorem 3.5 We first prove Theorem 3.5 for bounded and upper semicontinuous $G$ in the case where $\mathfrak{P}=\Omega$.

By Proposition 3.2 in the case where $\mathfrak{P}=\Omega$, absence of WFLVR is equivalent to $\mathcal{M}_{\mathcal{X}_{c}, \mathcal{P}, \Omega} \neq 0$, for which to hold Condition 3.1 is both necessary and sufficient. Following the classical arguments in Breeden and Litzenberger [7], by defining probability measures $\mu_{i}$ on $\mathbb{R}_{+}$via $\mu_{i}([0, K])=1+c_{i}^{\prime}(K)$ for $K \in \mathbb{R}_{+}$, we can encode the market prices $\mathcal{P}$, or $c_{i}(K)$, via $\mu_{i}$ with $c_{i}(K)=\mathcal{P}\left(\left(\mathbb{S}_{i}-K\right)^{+}\right)=\int(s-K)^{+} \mu_{i}(\mathrm{~d} s)$. Hence $\mathcal{M}_{\mathcal{X}_{c}, \mathcal{P}, \Omega}^{-}=\mathcal{M}_{\mu}^{-}$.

By Remark 2.3, to show (3.2), it suffices to show $V_{\mathcal{X}_{c}, \mathcal{P}, \Omega}(G) \leq P_{\mathcal{X}_{c}, \mathcal{P}, \Omega}(G)$. Define $G_{\Delta}: \mathbb{R}_{+}^{n} \rightarrow[-\infty, \infty)$ by

$$
G_{\Delta}(\mathbb{S}):=G(\mathbb{S})-\sum_{j=0}^{n-1} \Delta_{j}\left(\mathbb{S}_{1}, \ldots, \mathbb{S}_{j}\right)\left(\mathbb{S}_{j+1}-\mathbb{S}_{j}\right)
$$

It is clear that if $\Delta_{j} \in C_{c}\left(\mathbb{R}_{+}^{j}, \mathbb{R}_{+}\right)$for every $j$, then $G_{\Delta}(\mathbb{S})$ is upper semi-continuous and bounded, and hence satisfies (3.1). We can deduce that

$$
\begin{aligned}
V_{\mathcal{X}_{c}, \mathcal{P}, \Omega}(G) & =\inf _{(X, \Delta) \in \mathcal{A}_{c} \text { with } \Psi_{X, \Delta \geq G}} \mathcal{P}(X) \\
& \leq \inf _{\Delta_{j} \in C_{c}\left(\mathbb{R}_{+}^{j}, \mathbb{R}_{+}\right)} \inf \left\{\mathcal{P}(X): X \in \mathcal{A}_{c} \text { with } X \geq G_{\Delta} \text { on } \Omega\right\} \\
& =\inf _{\Delta_{j} \in C_{c}\left(\mathbb{R}_{+}^{j}, \mathbb{R}_{+}\right)} \sup _{\pi \in \Pi_{\mu}} \int_{\mathbb{R}_{+}^{n}} G_{\Delta}\left(s_{1}, \ldots, s_{n}\right) \mathrm{d} \pi\left(s_{1}, \ldots, s_{n}\right) \\
& =\sup _{\pi \in \Pi_{\mu} \Delta_{j} \in C_{c}\left(\mathbb{R}_{+}^{n}, \mathbb{R}_{+}\right)} \int_{\mathbb{R}_{+}^{n}} G_{, \Delta}\left(s_{1}, \ldots, s_{n}\right) \mathrm{d} \pi\left(s_{1}, \ldots, s_{n}\right),
\end{aligned}
$$


where the equality between (6.4) and (6.5) is guaranteed by Lemma 6.3. In order to justify the equality between (6.5) and (6.6), we apply the min-max theorem (see Terkelsen [42, Corollary 2]) to the compact convex set $\Pi_{\mu}$, the convex set $\mathbb{R}_{+} \times C_{c}\left(\mathbb{R}_{+}, \mathbb{R}_{+}\right) \times \cdots \times C_{c}\left(\mathbb{R}_{+}^{n-1}, \mathbb{R}_{+}\right)$, and the function

$$
f\left(\pi,\left(\Delta_{j}\right)\right)=\int_{\mathbb{R}_{+}^{n}} G_{\Delta}\left(s_{1}, \ldots, s_{n}\right) \mathrm{d} \pi\left(s_{1}, \ldots, s_{n}\right) .
$$

Clearly, $f$ is affine in each of the variables. Furthermore, by the Portmanteau theorem, $f\left(\cdot,\left(\Delta_{j}\right)\right)$ is upper semi-continuous on $\Pi_{\mu}$. Therefore, the assumptions of Corollary 2 in Terkelsen [42] are satisfied.

The last step is to establish the equality

$$
\sup _{\pi \in \Pi_{\mu} \Delta_{j} \in C_{c}\left(\mathbb{R}_{+}^{j}, \mathbb{R}_{+}\right)} \int_{\mathbb{R}_{+}^{n}} G_{\Delta}\left(s_{1}, \ldots, s_{n}\right) \mathrm{d} \pi\left(s_{1}, \ldots, s_{n}\right)=\sup _{\mathbb{P} \in \mathcal{M}_{\mu}^{-}} \mathbb{E}_{\mathbb{P}}[G(\mathbb{S})] .
$$

If $\pi \notin \mathcal{M}_{\mu}^{-}$, then by Lemma 6.1 , there is a $\Delta_{j} \in C_{c}\left(\mathbb{R}_{+}^{j}, \mathbb{R}_{+}\right)$for some $j$ such that

$$
B=\int_{\mathbb{R}_{+}^{n}} \Delta_{j}\left(s_{1}, \ldots, s_{j}\right)\left(s_{j+1}-s_{j}\right) \mathrm{d} \pi\left(s_{1}, \ldots, s_{n}\right)>0 .
$$

By scaling, $B$ can be arbitrarily large. Hence, if $\pi \notin \mathcal{M}_{\mu}^{-}$, then

$$
\inf _{\Delta_{j} \in C_{c}\left(\mathbb{R}_{+}^{j}, \mathbb{R}_{+}\right)} \int_{\mathbb{R}_{+}^{n}} G_{\Delta}\left(s_{1}, \ldots, s_{n}\right) \mathrm{d} \pi\left(s_{1}, \ldots, s_{n}\right)=-\infty .
$$

Since $G$ is bounded and $\mathcal{M}_{\mu}^{-} \neq \emptyset, V_{\mathcal{X}_{c}, \mathcal{P}}(G) \geq \sup _{\mathbb{P} \in \mathcal{M}_{\mu}^{-}} \mathbb{E}_{\mathbb{P}}[G]>-\infty$. Therefore, in the LHS of (6.7), it suffices to consider $\pi \in \mathcal{M}_{\mu}^{-}$and then

$$
\inf _{\Delta_{j} \in C_{c}\left(\mathbb{R}_{+}^{j}, \mathbb{R}_{+}\right)} \sum_{j=0}^{n-1} \int \Delta_{j}\left(s_{1}, \ldots, s_{j}\right)\left(s_{j}-s_{j+1}\right) \mathrm{d} \pi=0 .
$$

Hence

$$
\begin{aligned}
& \sup _{\pi \in \mathcal{M}_{\mu}^{-}} \inf _{\Delta_{j} \in C_{c}\left(\mathbb{R}_{+}^{j}, \mathbb{R}_{+}\right)} \int_{\mathbb{R}_{+}^{n}} G_{\Delta}\left(s_{1}, \ldots, s_{n}\right) \mathrm{d} \pi\left(s_{1}, \ldots, s_{n}\right) \\
& \leq \sup _{\mathbb{P} \in \mathcal{M}_{\mu}^{-}} \mathbb{E}_{\mathbb{P}}[G]+\sup _{\pi \in \mathcal{M}_{\mu}^{-} \Delta_{j} \in C_{c}\left(\mathbb{R}_{+}^{j}, \mathbb{R}_{+}\right)} \sum_{j=0}^{n-1} \int \Delta_{j}\left(s_{1}, \ldots, s_{j}\right)\left(s_{j}-s_{j+1}\right) \mathrm{d} \pi \\
& =\sup _{\mathbb{P}} \mathbb{E}_{\mathbb{P}}[G] .
\end{aligned}
$$

6.4 Completing the proof of Proposition 3.2

In this section, we complete the proof of Proposition 3.2 in the case where $\mathfrak{P} \subsetneq \Omega$. 
Step 5. "no WFLVR $\Longrightarrow \exists$ MCM".

It remains to argue that when $\mathfrak{P}$ is a closed subset of $\Omega$ such that $\mathfrak{P} \subsetneq \Omega$ and Condition 3.1 is satisfied, the non-existence of a market-calibrated model concentrated on $\mathfrak{P}$ implies the existence of a WFLVR. In fact, in this case, there is a robust uniformly strong arbitrage. Define a lower semi-continuous function $\lambda_{\mathfrak{P}}: \mathbb{R}_{+}^{n} \rightarrow \mathbb{R}$ by

$$
\lambda_{\mathfrak{P}}\left(s_{1}, \ldots, s_{n}\right)=\mathbb{1}_{\left\{\left(s_{1}, \ldots, s_{n}\right) \notin \mathfrak{P}\right\}} .
$$

Then we apply Theorem 3.5 to the prediction set $\Omega$ and $-\lambda_{\mathfrak{P}}$ and find that

$$
V_{\mathcal{X}_{c}, \mathcal{P}, \Omega}\left(-\lambda_{\mathfrak{P}}\right)=\sup _{\mathbb{P} \in \mathcal{M}_{\mathcal{X}_{c}, \mathcal{P}, \Omega}^{-}} \mathbb{E}_{\mathbb{P}}\left[-\lambda_{\mathfrak{P}}\right]=: \alpha .
$$

If $\alpha=0$, then there exists a sequence $\left(\mathbb{P}_{k}\right)$ in $\mathcal{M}_{\mathcal{X}_{c}, \mathcal{P}, \Omega}^{-}$such that $\mathbb{P}_{k}\left[\mathfrak{P}^{c}\right] \rightarrow 0$. By Lemma $6.2, \mathcal{M}_{\mathcal{X}_{c}, \mathcal{P}, \Omega}^{-}$is compact and closed. Hence $\left(\mathbb{P}_{k}\right)_{k \in \mathbb{N}}$ has a subsequence converging to some $\mathbb{P} \in \mathcal{M}_{\mathcal{X}_{c}, \mathcal{P}, \Omega}^{-}$. In fact, by weak convergence of measures, $\mathbb{P}\left[\mathfrak{P}^{c}\right]=0$ and hence $\mathbb{P} \in \mathcal{M}_{\mathcal{X}}^{-}, \mathcal{P}, \mathfrak{P}$. This shows that the absence of a market-calibrated model concentrated on $\mathfrak{P}$ implies $\alpha<0$ and therefore the existence of a robust uniformly strong arbitrage (and hence WFLVR). Together with the results of Sect. 6.2, this completes the proof of Proposition 3.2.

With the proof of Proposition 3.2 established, we are now able to give a proof of Theorem 3.5 in the general case where $\mathfrak{P} \neq \Omega$.

\subsection{Completing the proof of Theorems 3.5 and 4.3: case where $\mathfrak{P} \subseteq \Omega$}

We now complete the proof of Theorems 3.5 and 4.3 for $G$ satisfying (3.1) in the case where $\mathfrak{P} \subseteq \Omega$. Again, since they are virtually identical, we only give the proof of Theorem 3.5 here.

If (3.2) holds for $G$, then (3.2) is still true for any function $\tilde{G}=G+X$ with $X$ of the form $a_{0}+\sum_{i=1}^{n} a_{i}\left(S_{i}-K_{i}\right)^{+}$. Therefore, without loss of generality, we may and do assume that $G$ is bounded from above.

Recall from (6.8) that $\lambda_{\mathfrak{P}}\left(s_{1}, \ldots, s_{n}\right)=\mathbb{1}_{\left\{\left(s_{1}, \ldots, s_{n}\right) \notin \mathfrak{P}\right\}}$ is bounded and lower semicontinuous and hence $G \vee(-N)-N \lambda_{\mathfrak{P}}$ is bounded and upper semi-continuous for each $N \in \mathbb{N}$. We also notice that

$$
V_{\mathcal{X}_{c}, \mathcal{P}, \mathfrak{P}}(G) \leq V_{\mathcal{X}_{c}, \mathcal{P}, \Omega}\left(G \vee(-N)-N \lambda_{\mathfrak{P}}\right)
$$

for each $N \in \mathbb{N}$, since any superreplicating portfolio of $G \vee(-N)-N \lambda_{\mathfrak{P}}$ on $\Omega$ naturally superreplicates $G$ on $\mathfrak{P}$. Thus

$$
\begin{aligned}
V_{\mathcal{X}_{c}, \mathcal{P}, \mathfrak{P}}(G) & \leq \inf _{N \geq 0} V_{\mathcal{X}_{c}, \mathcal{P}, \Omega}\left(G \vee(-N)-N \lambda_{\mathfrak{P}}\right) \\
& =\inf _{N \geq 0} P_{\mathcal{X}_{c}, \mathcal{P}, \Omega}\left(G \vee(-N)-N \lambda_{\mathfrak{P}}\right) \\
& =\inf _{N \geq 0} \sup _{\mathbb{P} \in \mathcal{M}_{\mu}^{-}} \mathbb{E}_{\mathbb{P}}\left[G \vee(-N)-N \lambda_{\mathfrak{P}}\right] .
\end{aligned}
$$


Define $f_{N}: \mathcal{M}_{\mu}^{-} \rightarrow(-\infty, \infty)$ by $f_{N}(\mathbb{P})=\mathbb{E}_{\mathbb{P}}\left[G \vee(-N)-N \lambda_{\mathfrak{P}}\right]$. Note that $f_{N}$ is upper semi-continuous on $\mathcal{M}_{\mu}^{-}$and $f_{N} \geq f_{N+1}$ for every $N \in \mathbb{N}$. Hence, applying the min-max theorem (see Terkelsen [42, Corollary 1]) to the compact convex set $\mathcal{M}_{\mu}^{-}$and $\left(f_{N}\right)_{N \in \mathbb{N}}$, we have

$$
\inf _{N \geq 0} \sup _{\mathbb{P} \in \mathcal{M}_{\mu}^{-}} \mathbb{E}_{\mathbb{P}}\left[G \vee(-N)-N \lambda_{\mathfrak{P}}\right]=\sup _{\mathbb{P} \in \mathcal{M}_{\mu}^{-}} \inf _{N \geq 0} \mathbb{E}_{\mathbb{P}}\left[G \vee(-N)-N \lambda_{\mathfrak{P}}\right]
$$

Define $G_{\mathfrak{P}}$ by $G_{\mathfrak{P}}=G$ on $\mathfrak{P}$ and $-\infty$ elsewhere. Note that $G_{\mathfrak{P}}$ is the pointwise limit of $G \vee(-N)-N \lambda_{\mathfrak{P}}$ as $N \rightarrow \infty$. Then by Fatou's lemma,

$$
\sup _{\mathbb{P} \in \mathcal{M}_{\mu}^{-}} \inf _{N \geq 0} \mathbb{E}_{\mathbb{P}}\left[G \vee(-N)-N \lambda_{\mathfrak{P}}\right] \leq \sup _{\mathbb{P} \in \mathcal{M}_{\mu}^{-}} \mathbb{E}_{\mathbb{P}}\left[G_{\mathfrak{P}}\right]=\sup _{\mathbb{P} \in \mathcal{M}_{\boldsymbol{\mu}, \mathfrak{P}}^{-}} \mathbb{E}_{\mathbb{P}}[G]
$$

Therefore, we have $V_{\mathcal{X}_{c}, \mathcal{P}, \mathfrak{P}}(G) \leq P_{\mathcal{X}_{c}, \mathcal{P}, \mathfrak{P}}(G)$, which together with Remark 2.3 leads us to conclude that

$$
V_{\mathcal{X}_{c}, \mathcal{P}, \mathfrak{P}}(G)=P_{\mathcal{X}_{c}, \mathcal{P}, \mathfrak{P}}(G)
$$

\subsection{Proof of Theorem 4.5}

Proof Given a semi-static superreplicating strategy $(X, \Delta)$, we have by definition for any $\left(s_{1}, \ldots, s_{n}\right) \in \mathfrak{P}$ that

$$
X\left(s_{1}, \ldots, s_{n}\right)+\sum_{i=0}^{n-1} \Delta_{i}\left(s_{1}, \ldots, s_{i}\right)\left(s_{i+1}-s_{i}\right) \geq G\left(s_{1}, \ldots, s_{n}\right) .
$$

We start with the following

Claim If $(X, \Delta)$ is a semi-static superreplicating strategy of $G$ on the prediction set $\mathfrak{P}$, then $\Delta_{j} \geq \beta_{j}$ for any $i=0, \ldots, n-1$.

We prove the claim by induction. When $j=n-1$, we fix $s_{n-1}:=\left(s_{1}, \ldots, s_{n-1}\right)$. Letting $s_{n} \in \mathfrak{P}\left(s_{n-1}, n\right):=\left\{x:\left(s_{1}, \ldots, s_{n-1}, x\right) \in \mathfrak{P}\right\}$ go to infinity, it follows from (6.9) that

$$
\Delta_{n-1}\left(s_{1}, \ldots, s_{n-1}\right) \geq \limsup _{x \rightarrow \infty, x \in \mathfrak{P}\left(s_{n-1}, n\right)} \frac{G\left(s_{1}, \ldots, s_{n-1}, x\right)}{x} .
$$

This, together with $\Delta_{n-1} \geq 0$, yields

$$
\begin{aligned}
\Delta_{n-1}\left(s_{1}, \ldots, s_{n-1}\right) & \geq \limsup _{x \rightarrow \infty}\left(\frac{G\left(s_{1}, \ldots, s_{n-1}, x\right)}{x} \mathbb{1}_{\mathfrak{P}}\left(s_{1}, \ldots, s_{n-1}, x\right)\right) \vee 0 \\
& =\beta_{n-1}\left(s_{1}, \ldots, s_{n-1}\right) .
\end{aligned}
$$


Now suppose the claim holds for $j=i+1$ with $i \leq n-2$. We fix a vector $\boldsymbol{s}_{n-1}:=\left(s_{1}, \ldots, s_{i}, s_{i+2}, \ldots, s_{n}\right)$ and denote

$$
\mathfrak{P}\left(s_{n-1}, i+1\right):=\left\{x:\left(s_{1}, \ldots, s_{i}, x, s_{i+2}, \ldots, s_{n}\right) \in \mathfrak{P}\right\} .
$$

If $\mathfrak{P}\left(\boldsymbol{s}_{n-1}, i+1\right)$ is unbounded, then by taking $x \in \mathfrak{P}\left(\boldsymbol{s}_{n-1}, i+1\right)$ to infinity, (6.9) implies

$$
\begin{aligned}
\Delta_{i}\left(s_{1}, \ldots, s_{i}\right) \geq \limsup _{x \rightarrow \infty, x \in \mathfrak{P}\left(s_{n-1}, i+1\right)} & \left(\Delta_{i+1}\left(s_{1}, \ldots, s_{i}, x\right)\right. \\
& \left.+\frac{G\left(s_{1}, \ldots, s_{i}, x, s_{i+2}, \ldots, s_{n}\right)}{x}\right) .
\end{aligned}
$$

If the right-hand side is nonnegative, then

$$
\begin{aligned}
& \limsup _{x \rightarrow \infty, x \in \mathfrak{P}\left(s_{n-1}, i+1\right)}\left(\Delta_{i+1}\left(s_{1}, \ldots, s_{i}, x\right)+\frac{G\left(s_{1}, \ldots, s_{i}, x, s_{i+2}, \ldots, s_{n}\right)}{x}\right) \\
&=\limsup _{x \rightarrow \infty}\left(\mathbb{1}_{\mathfrak{P}}\left(s_{1}, \ldots, s_{i}, x, s_{i+2}, \ldots, s_{n}\right)\right. \\
&\left.\times\left(\Delta_{i+1}\left(s_{1}, \ldots, s_{i}, x\right)+\frac{G\left(s_{1}, \ldots, s_{i}, x, s_{i+2}, \ldots, s_{n}\right)}{x}\right)\right) .
\end{aligned}
$$

Hence, as $\Delta_{i} \geq 0$, when $\mathfrak{P}\left(s_{n-1}, i+1\right)$ is unbounded, we have

$$
\begin{aligned}
\Delta_{i}\left(s_{1}, \ldots, s_{i}\right) & \\
\geq \limsup _{x \rightarrow \infty}( & \left(\Delta_{i+1}\left(s_{1}, \ldots, s_{i}, x\right)+\frac{G\left(s_{1}, \ldots, s_{i}, x, s_{i+2}, \ldots, s_{n}\right)}{x}\right) \\
& \left.\times \mathbb{1}_{\mathfrak{P}}\left(s_{1}, \ldots, s_{i}, x, s_{i+2}, \ldots, s_{n}\right)\right) \vee 0 \\
\geq \limsup _{x \rightarrow \infty}( & \left(\beta_{i+1}\left(s_{1}, \ldots, s_{i}, x\right)+\frac{G\left(s_{1}, \ldots, s_{i}, x, s_{i+2}, \ldots, s_{n}\right)}{x}\right) \\
& \left.\times \mathbb{1}_{\mathfrak{P}}\left(s_{1}, \ldots, s_{i}, x, s_{i+2}, \ldots, s_{n}\right)\right) \vee 0 .
\end{aligned}
$$

On the other hand, when $\mathfrak{P}\left(\boldsymbol{s}_{n-1}, i+1\right)$ is bounded, we notice that

$$
\begin{aligned}
\limsup _{x \rightarrow \infty}( & \left(\beta_{i+1}\left(s_{1}, \ldots, s_{i}, x\right)+\frac{G\left(s_{1}, \ldots, s_{i}, x, s_{i+2}, \ldots, s_{n}\right)}{x}\right) \\
& \left.\times \mathbb{1}_{\mathfrak{P}}\left(s_{1}, \ldots, s_{i}, x, s_{i+2}, \ldots, s_{n}\right)\right)=0 .
\end{aligned}
$$


Hence, the inequality in (6.10) is true in either case. In addition, as it holds for all $s_{1}, \ldots, s_{i}, s_{i+2}, \ldots, s_{n} \in \mathbb{R}_{+}$, we can conclude that

$$
\begin{aligned}
& \Delta_{i}\left(s_{1}, \ldots, s_{i}\right) \\
& \geq \sup _{s_{i+2}, \ldots, s_{n} \in \mathbb{R}_{+}} \limsup _{x \rightarrow \infty}\left(\left(\Delta_{i+1}\left(s_{1}, \ldots, s_{i}, x\right)+\frac{G\left(s_{1}, \ldots, s_{i}, x, s_{i+2}, \ldots, s_{n}\right)}{x}\right)\right. \\
& \left.\times \mathbb{1}_{\mathfrak{P}}\left(s_{1}, \ldots, s_{i}, x, s_{i+2}, \ldots, s_{n}\right)\right) \vee 0 \\
& =\beta_{i}\left(s_{1}, \ldots, s_{i}\right) \quad \text { for any } s_{1}, \ldots, s_{i} \in \mathbb{R}_{+} \text {. }
\end{aligned}
$$

This ends the induction and the proof of the claim.

It follows from the claim above that for any $(X, \Delta) \in \mathcal{A}_{p}$ that superreplicates $G$ on $\mathfrak{P}$ and any $\mathbb{P} \in \mathcal{M}_{\boldsymbol{\mu}, \mathfrak{P}}^{-}$, we have

$$
\begin{aligned}
\mathbb{E}_{\mathbb{P}}[G] & \leq \mathbb{E}_{\mathbb{P}}\left[X(\mathbb{S})+\sum_{i=0}^{n-1} \Delta_{i}\left(\mathbb{S}_{1}, \ldots, \mathbb{S}_{i}\right)\left(\mathbb{S}_{i+1}-\mathbb{S}_{i}\right)\right] \\
& \leq \mathbb{E}_{\mathbb{P}}\left[X(\mathbb{S})+\sum_{i=0}^{n-1} \beta_{i}\left(\mathbb{S}_{1}, \ldots, \mathbb{S}_{i}\right)\left(\mathbb{S}_{i+1}-\mathbb{S}_{i}\right)\right]
\end{aligned}
$$

which implies that

$$
V_{\mu, \mathfrak{P}}^{(p)}(G) \geq \sup _{\mathbb{P} \in \mathcal{M}_{\mu, \mathfrak{P}}^{-}} \mathbb{E}_{\mathbb{P}}\left[G-\sum_{i=0}^{n-1} \beta_{i}\left(\mathbb{S}_{1}, \ldots, \mathbb{S}_{i}\right)\left(\mathbb{S}_{i+1}-\mathbb{S}_{i}\right)\right]
$$

For the converse inequality, we let $\mathcal{B}_{b}\left(\mathbb{R}_{+}^{d}, \mathbb{R}_{+}\right)$be the set of bounded measurable functions $f: \mathbb{R}_{+}^{d} \rightarrow \mathbb{R}_{+}$and denote by $\mathcal{Z}$ the collection of all tuples of functions $\left(\Delta_{j}\right)_{j=0}^{n-1} \in \mathbb{R}_{+} \times \mathcal{B}_{b}\left(\mathbb{R}_{+}, \mathbb{R}_{+}\right) \times \cdots \times \mathcal{B}_{b}\left(\mathbb{R}_{+}^{n-1}, \mathbb{R}_{+}\right)$such that

$$
G_{\Delta}(\mathbb{S}):=G(\mathbb{S})-\sum_{i=0}^{n-1} \Delta_{i}\left(\mathbb{S}_{1}, \ldots, \mathbb{S}_{i}\right)\left(\mathbb{S}_{i+1}-\mathbb{S}_{i}\right)
$$

is upper semi-continuous and bounded from above on $\mathfrak{P}$. Note that $\mathcal{Z}$ is a convex subset of $\mathbb{R}_{+} \times \mathcal{B}_{b}\left(\mathbb{R}_{+}, \mathbb{R}_{+}\right) \times \cdots \times \mathcal{B}_{b}\left(\mathbb{R}_{+}^{n-1}, \mathbb{R}_{+}\right)$. Then we can apply the min-max theorem from Terkelsen [42, Corollary 2] to the compact convex set $\mathcal{M}_{\boldsymbol{\mu}, \mathfrak{P}}^{-}, \mathcal{Z}$ and the function

$$
f\left(\pi,\left(\Delta_{j}\right)\right)=\int\left(G\left(s_{1}, \ldots, s_{n}\right)-\sum_{i=0}^{n-1} \Delta_{i}\left(s_{1}, \ldots, s_{i}\right)\left(s_{i+1}-s_{i}\right)\right) \mathrm{d} \pi\left(s_{1}, \ldots, s_{n}\right) .
$$

Clearly, $f$ is affine in each variable and by the Portmanteau theorem $f\left(\cdot,\left(\Delta_{j}\right)\right)$ is upper semi-continuous on $\mathcal{M}_{\boldsymbol{\mu}, \mathfrak{P}}^{-}$. So the assumptions of Corollary 2 in Terkelsen 
[42] are satisfied, and we find that

$$
\begin{aligned}
& V_{\mu, \mathfrak{P}}^{(p)}(G)=\inf \left\{\mathcal{P}(X):(X, \Delta) \in \mathcal{A}_{p} \text { with } \Psi_{X, \Delta} \geq G \text { on } \mathfrak{P}\right\} \\
& \leq \inf _{\Delta \in \mathcal{Z}} \inf \left\{\mathcal{P}(X):(X, \tilde{\Delta}) \in \mathcal{A}_{p} \text { with } \Psi_{X, \Delta+\tilde{\Delta}} \geq G \text { on } \mathfrak{P}\right\} \\
& =\inf _{\Delta \in \mathcal{Z}} \inf \left\{\mathcal{P}(X):(X, \tilde{\Delta}) \in \mathcal{A}_{p} \text { with } \Psi_{X, \tilde{\Delta}} \geq G_{\Delta} \text { on } \mathfrak{P}\right\}
\end{aligned}
$$

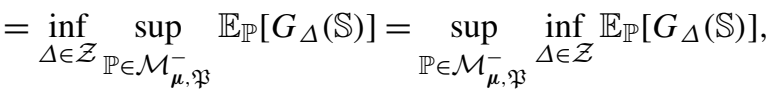

where the inequality between (6.11) and (6.12) is by restricting the delta-hedging terms to a smaller set, and the equality between (6.12) and (6.13) follows from Theorem 4.3.

To conclude, from the assumption we know there exists a sequence $\left(\beta^{(N)}\right)$ in $\mathcal{Z}$ such that $G_{\beta^{(N)}}(\mathbb{S})=G(\mathbb{S})-\sum_{i=0}^{n-1} \beta_{i}^{(N)}\left(\mathbb{S}_{1}, \ldots, \mathbb{S}_{i}\right)\left(\mathbb{S}_{i+1}-\mathbb{S}_{i}\right)$ is upper semicontinuous, bounded from above on $\mathfrak{P}$ and $G_{\beta^{(N)}} \rightarrow G_{\beta}$ pointwise as $N \rightarrow \infty$. Hence, by Fatou's lemma,

$$
\limsup _{N \rightarrow \infty} \mathbb{E}_{\mathbb{P}}\left[G_{\beta^{(N)}}(\mathbb{S})\right] \leq \mathbb{E}_{\mathbb{P}}\left[G-\sum_{i=0}^{n-1} \beta_{i}\left(\mathbb{S}_{1}, \ldots, \mathbb{S}_{i}\right)\left(\mathbb{S}_{i+1}-\mathbb{S}_{i}\right)\right]
$$

holds for any $\mathbb{P} \in \mathcal{M}_{\boldsymbol{\mu}, \mathfrak{P}}^{-}$, and therefore we have

$$
\sup _{\mathbb{P} \in \mathcal{M}_{\mu, \mathfrak{P}}^{-}} \inf _{\Delta \in \mathcal{Z}} \mathbb{E}_{\mathbb{P}}\left[G_{\Delta}(\mathbb{S})\right] \leq \sup _{\mathbb{P} \in \mathcal{M}_{\mu, \mathfrak{P}}^{-}} \mathbb{E}_{\mathbb{P}}\left[G-\sum_{i=0}^{n-1} \beta_{i}\left(\mathbb{S}_{1}, \ldots, \mathbb{S}_{i}\right)\left(\mathbb{S}_{i+1}-\mathbb{S}_{i}\right)\right]
$$

which leads us to conclude that

$$
V_{\mu, \mathfrak{P}}^{(p)}(G) \leq \sup _{\mathbb{P} \in \mathcal{M}_{\mu, \mathfrak{P}}^{-}} \mathbb{E}_{\mathbb{P}}\left[G-\sum_{i=0}^{n-1} \beta_{i}\left(\mathbb{S}_{1}, \ldots, \mathbb{S}_{i}\right)\left(\mathbb{S}_{i+1}-\mathbb{S}_{i}\right)\right]
$$

\section{References}

1. Acciaio, B., Beiglböck, M., Penkner, F., Schachermayer, W.: A model-free version of the fundamental theorem of asset pricing and the super-replication theorem. Math. Finance 26, 233-251 (2016). Available at http://onlinelibrary.wiley.com/doi/10.1111/mafi.12060/full

2. Battalio, R., Schultz, P.: Regulatory uncertainty and market liquidity: the 2008 short sale ban's impact on equity option markets. J. Finance 66, 2013-2053 (2011)

3. Bayraktar, E., Zhou, Z.: On arbitrage and duality under model uncertainty and portfolio constraints. Math. Finance (2015, electronic). Available at http://onlinelibrary.wiley.com/doi/10.1111/ mafi.12104/full

4. Beiglböck, M., Henry-Labordère, P., Penkner, F.: Model-independent bounds for option prices: a mass transport approach. Finance Stoch. 17, 477-501 (2013)

5. Black, F., Scholes, M.: The pricing of options and corporate liabilities. J. Polit. Econ. 81, 637-654 (1973) 
6. Bouchard, B., Nutz, M.: Arbitrage and duality in nondominated discrete-time models. Ann. Appl. Probab. 25, 823-859 (2015)

7. Breeden, D.T., Litzenberger, R.H.: Prices of state-contingent claims implicit in option prices. J. Bus. 5, 621-651 (1978)

8. Bruckner, A.M., Thomson, B.S., Bruckner, J.B.: Real Analysis. Prentice Hall, New York (2008)

9. Carr, P., Fisher, T., Ruf, J.: On the hedging of options on exploding exchange rates. Finance Stoch. 18, 115-144 (2014)

10. Cox, A.M.G., Hobson, D.G.: Local martingales, bubbles and option prices. Finance Stoch. 9, 477-492 (2005)

11. Cox, A.M.G., Obłój, J.: Robust pricing and hedging of double no-touch options. Finance Stoch. 15, 573-605 (2011)

12. Cvitanić, J., Karatzas, I.: Hedging contingent claims with constrained portfolios. Ann. Appl. Probab. 3, 652-681 (1993)

13. Cvitanić, J., Pham, H., Touzi, N.: Super-replication in stochastic volatility models under portfolio constraints. J. Appl. Probab. 36, 523-545 (1999)

14. Davis, M.H.A., Hobson, D.G.: The range of traded option prices. Math. Finance 17, 1-14 (2007)

15. Delbaen, F., Schachermayer, W.: A general version of the fundamental theorem of asset pricing. Math. Ann. 300, 463-520 (1994)

16. Delbaen, F., Schachermayer, W.: Arbitrage and free lunch with bounded risk for unbounded continuous processes. Math. Finance 4, 343-348 (1994)

17. El Karoui, N., Quenez, M.-C.: Dynamic programming and pricing of contingent claims in an incomplete market. SIAM J. Control Optim. 33, 29-66 (1995)

18. Fahim, A., Huang, Y.: Model-independent superhedging under portfolio constraints. Finance Stoch. 20, 51-81 (2016)

19. Guasoni, P., Rasonyi, M.: Fragility of arbitrage and bubbles in local martingale diffusion models. Finance Stoch. 19, 215-231 (2015)

20. Harrison, J.M., Kreps, D.M.: Speculative investor behavior in a stock market with heterogeneous expectations. Q. J. Econ. 92, 323-336 (1978)

21. Hendershott, T., Namvar, E., Phillips, B.: The intended and collateral effects of short-sale bans as a regulatory tool. J. Invest. Manag. 11(3), 5-13 (2013)

22. Herdegen, M., Schweizer, M.: Strong bubbles and strict local martingales. Int. J. Theor. Appl. Finance, to appear. Available at http://papers.ssrn.com/sol3/papers.cfm?abstract_id $=2566815$

23. Heston, S.L., Loewenstein, M., Willard, G.A.: Options and bubbles. Rev. Financ. Stud. 20, 359-390 (2007)

24. Hobson, D.G.: Robust hedging of the lookback option. Finance Stoch. 2, 329-347 (1998)

25. Hou, Z., Obłój, J.: On robust pricing-hedging duality in continuous time. Working paper (2015). Available at http://arxiv.org/abs/1503.02822

26. Hugonnier, J.: Rational asset pricing bubbles and portfolio constraints. J. Econ. Theory 147, 2260$2302(2012)$

27. Jarrow, R., Protter, P.: Discrete versus continuous time models: local martingales and singular processes in asset pricing theory. Finance Res. Lett. 9, 58-62 (2012)

28. Jarrow, R., Protter, P., Shimbo, K.: Asset price bubbles in complete markets. In: Fu, M.C., et al. (eds.) Advances in Mathematical Finance. Applied and Numerical Harmonic Analysis, pp. 97-121. Birkhäuser, Boston (2007)

29. Jarrow, R.A., Protter, P., Shimbo, K.: Asset price bubbles in incomplete markets. Math. Finance 20, $145-185$ (2010)

30. Jouini, E., Kallal, H.: Arbitrage in securities markets with short-sales constraints. Math. Finance 5, 197-232 (1995)

31. Lewis, A.L.: Option Valuation Under Stochastic Volatility: With Mathematica Code. Finance Press, California (2000)

32. Loewenstein, M., Willard, G.A.: Local martingales, arbitrage, and viability. Free snacks and cheap thrills. Econ. Theory 16, 135-161 (2000)

33. Merton, R.C.: Theory of rational option pricing. Bell J. Econ. 4, 141-183 (1973)

34. Mykland, P.A.: Financial options and statistical prediction intervals. Ann. Stat. 31, 1413-1438 (2003)

35. Pham, H., Touzi, N.: The fundamental theorem of asset pricing with cone constraints. J. Math. Econ. 31, 265-279 (1999)

36. Protter, P.: A mathematical theory of financial bubbles. In: Henderson, V., Sircar, R. (eds.) ParisPrinceton Lectures on Mathematical Finance 2013. Lecture Notes in Mathematics, vol. 2081, pp. 1108. Springer, Berlin (2013) 
37. Pulido, S.: The fundamental theorem of asset pricing, the hedging problem and maximal claims in financial markets with short sales prohibitions. Ann. Appl. Probab. 24, 54-75 (2014)

38. Scheinkman, J.A., Xiong, W.: Overconfidence and speculative bubbles. J. Polit. Econ. 111, 11831220 (2003)

39. Sin, C.A.: Complications with stochastic volatility models. Adv. Appl. Probab. 30, 256-268 (1998)

40. Spoida, P.: Robust pricing and hedging beyond one marginal. D.Phil. Thesis, University of Oxford (2014). Available at http://ora.ox.ac.uk/objects/uuid:0315824b-52f7-4e44-9ac6-0a688c49762c

41. Strassen, V.: The existence of probability measures with given marginals. Ann. Math. Stat. 36, 423439 (1965)

42. Terkelsen, F.: Some minimax theorems. Math. Scand. 31, 405-413 (1972) 